\title{
ALTERNATING SEQUENCES AND INDUCED OPERATORS
}

\author{
M. A. AKCOGLU AND R. E. BRADLEY
}

\begin{abstract}
We show that when a positive $L_{p}$ contraction is equipped with a norming function having full support, then it is related in a natural way to an operator on any other $L_{p}$ space, $1<p<\infty$. This construction is used to generalize a theorem of Rota concerning the convergence of alternating sequences.
\end{abstract}

\section{INTRODUCTION}

Let $L_{p}$ be the usual Banach space of complex-valued functions. Denote by $L_{p}^{+}$the class of $L_{p}$ functions taking nonnegative values. An $L_{p}$ operator $T$ is positive if $T L_{p}^{+} \subseteq L_{p}^{+}$. It is a contraction if $\|T f\|_{p} \leq\|f\|_{p}$ for every $f \in L_{p}$. We say $u$ is semi-invariant for a positive $L_{p}$ contraction $T$ if both $u$ and $T u$ have full support and $\|T u\|_{p}=\|u\|_{p}$.

(1.1) Theorem. Suppose $1<p<\infty$ and $1<r<\infty$. If $T$ is a positive $L_{p}$ contraction with a semi-invariant function $u$, then the formula

$$
T_{r} f=(T u)^{p / r-1} T\left(u^{1-p / r} f\right),
$$

where $f \in L_{r}$, defines a positive $L_{r}$ contraction. This operator is independent of the choice of semi-invariant function. We call $T_{r}$ the $L_{r}$ operator induced by $T$.

We apply this notion of induced operators to the question of convergence of alternating sequences. For simplicity of notation, the following theorem is stated for $L_{p}^{+}$only. The analogous result is proved for all of $L_{p} \cdot T^{*}$ denotes the adjoint of $T$; it is an operator on $L_{q}$ where $q=p(p-1)^{-1}$. Whenever $u$ is semi-invariant for an $L_{p}$ operator $T$, then $(T u)^{p-1}$ is semi-invariant for $T^{*}$.

(1.2) Theorem. Suppose $1<p<\infty$ and $1<r<\infty$. Let $\left\langle T_{n}\right\rangle_{n=1}^{\infty}$ be a sequence of positive $L_{p}$ contradictions with semi-invariant functions defined over a $\sigma$-finite

Received by the editors May 8, 1989.

1980 Mathematics Subject Classification (1985 Revision). Primary 28D99, 47A35.

Key words and phrases. Positive contractions of $L_{p}$, alternating sequences, maximal inequalities.

The research of the first author was supported in part by NSERC Grant A3974.

Some results in this paper appear in the second author's doctoral dissertation. Research supported in part by the Department of Mathematics, University of Toronto. 
Lebesgue space. Then

$$
\left(T_{1}^{*}\right)_{r} \cdots\left(T_{n}^{*}\right)_{r}\left(T_{n} \cdots T_{1} f\right)^{p / r}
$$

converges a.e. for every $f \in L_{p}^{+}$.

This theorem generalizes Rota's theorem of the alternating procedure [Rt]. We say an operator is bistochastic if $T \mathbf{1}=T^{*} \mathbf{1}=\mathbf{1}$, where $\mathbf{1}$ is the function taking the value 1 everywhere.

(1.3) Theorem (Rota). If $\left\langle T_{n}\right\rangle_{n=1}^{\infty}$ is a sequence of positive bistochastic operators over a probability space, then

$$
T_{1}^{*} \cdots T_{n}^{*} T_{n} \cdots T_{1} f
$$

converges a.e. for every $f \in L_{p}$, where $1<p<\infty$.

A positive bistochastic operator is a contraction of every $L_{p}$, where $1 \leq p \leq$ $\infty$; thus the expression (1.4) is well defined for every $p$. A positive $L_{p}$ contraction with a semi-invariant function does not necessarily have this property, but we may use the operator induced by $T^{*}$ to define a "pseudo-adjoint" of $T$ which operates on $L_{p}$.

In the finite measure case, $\mathbf{1}$ is semi-invariant for any bistochastic operator and for its adjoint. Furthermore, $T_{r}^{*}=T^{*}$ for any $r, 1<r<\infty$. Thus, Rota's theorem is a consequence of (1.1) with $r=p$.

\section{Preliminaries}

(2.1) Definitions. For any $\sigma$-finite measure space $(X, \mathscr{F}, \mu)$, let $\mathscr{M}(d \mu)$ be the vector space of $\mathscr{F}$-measurable complex-valued functions defined on $X$. Let $\mathscr{M}^{+}(d \mu)$ be the class of functions in $\mathscr{M}(d \mu)$ whose ranges are subsets of $\mathbb{R}^{+}=[0, \infty)$. Let $\overline{\mathscr{M}}^{+}(d \mu)$ be the set of $\mathscr{F}$-measurable functions on $X$ with values in the extended nonnegative reals, $[0, \infty]$.

The usual Banach space of functions in $\mathscr{M}(d \mu)$ for which $\int_{X}|f|^{p} d \mu<\infty$ is denoted by $L_{p}(d \mu)$, where $1 \leq p<\infty$, while $L_{\infty}(d \mu)$ denotes the space of essentially bounded functions $\mathscr{M}(d \mu)$. We also use $L_{p}^{+}(d \mu)=L_{p}(d \mu) \cap$ $\mathscr{M}^{+}(d \mu)$. All of the relations between the functions in these classes are in the $\mu$-a.e. sense, even when this is not made explicit. With the convention $0 \cdot \infty=0$, functions in $\overline{\mathscr{M}}^{+}(d \mu)$ may be multiplied pointwise.

Let $(Y, \mathscr{G}, \nu)$ be another $\sigma$-finite measure space. Consider the class of all mappings

$$
T: \overline{\mathscr{M}}^{+}(d \mu) \rightarrow \overline{\mathscr{M}}^{+}(d \nu)
$$

which satisfy the following two conditions:

(2.2) $T$ is "positive-linear"; that is, if $\alpha, \beta \in \mathbb{R}^{+}$and $f, g \in \overline{\mathscr{M}}^{+}(d \mu)$, then

$$
T(\alpha f+\beta g)=\alpha T f+\beta T g .
$$


(2.3) $T$ is "order-continuous" in the sense that $T f_{n} \uparrow T f \nu$-a.e. whenever $f_{n} \uparrow$ $f \mu$-a.e. (the arrows indicate monotone nondecreasing pointwise convergence in $\mathbb{R}^{+}$).

If $T$ is such a mapping, then its restriction to $\mathscr{M}^{+}(d \mu)$ need not be extendable linearly to $\mathscr{M}(d \mu)$. Thus, these mappings should not necessarily be associated with the usual class of linear operators. Nonetheless, it is convenient to make the following definition.

(2.4) Definition A mapping satisfying (2.2) and (2.3) will be called a positive operator on $\overline{\mathscr{M}}^{+}(d \mu)$ (or from $\overline{\mathscr{M}}^{+}(d \mu)$ to $\overline{\mathscr{M}}^{+}(d \nu)$ ).

(2.5) Lemma. Given a positive operator $T: \overline{\mathscr{M}}^{+}(d \mu) \rightarrow \overline{\mathscr{M}}^{+}(d \nu)$ there exists a unique positive operator $T^{*}: \overline{\mathscr{M}}^{+}(d \nu) \rightarrow \overline{\mathscr{M}}^{+}(d \mu)$ such that

$$
\int_{X} f T^{*} g d \mu=\int_{Y} T f \cdot g d \nu
$$

for every $f \in \overline{\mathscr{M}}^{+}(d \mu)$ and $g \in \overline{\mathscr{M}}^{+}(d \nu)$.

Proof. Given $g \in \overline{\mathscr{M}}^{+}(d \nu)$, the mapping

$$
f \in \overline{\mathscr{M}}^{+}(d \mu) \mapsto \int_{Y} T f \cdot g d \nu \in \mathbb{R}^{+}
$$

is integration with respect to some measure on $(X, \mathscr{F})$ which is absolutely continuous with respect to $\mu$. This measure may be represented as $\rho d \mu$ for some $\rho \in \overline{\mathscr{M}}^{+}(d \mu)$. Define $T^{*}$ by $T^{*} g=\rho$.

(2.6) Definition. The operator $T^{*}$ defined above is called the adjoint of $T$.

If $T: L_{p}(d \mu) \rightarrow L_{p}(d \nu)$ is a positive operator in the usual sense, then its restriction to $L_{p}^{+}(d \mu)$ can be extended to a positive operator on $\overline{\mathscr{M}}^{+}(d \mu)$, which will also be called $T$. It is unique by the requirement that it satisfy (2.3). If a positive operator on $\overline{\mathscr{M}}^{+}(d \mu)$ in the sense of (2.4) can be obtained in this way, then we will call it a positive $L_{p}$ operator on $\overline{\mathscr{M}}^{+}(d \mu)$. The following definition states this in a different way.

(2.7) Definition. A positive operator $T$ on $\overline{\mathscr{M}}^{+}(d \mu)$ is said to be a positive $L_{p}$ operator if

$$
\|T\|_{p}^{p}=\sup \left\{\int(T f)^{p} d \nu \mid f \in \overline{\mathscr{M}}^{+}(d \mu) \text { and } \int f^{p} d \mu \leq 1\right\}
$$

is finite. If, furthermore, $\|T\|_{p} \leq 1$, then $T$ is called a positive $L_{p}$ contraction.

Throughout this paper, whenever a number $p$ with $1<p<\infty$ is understood, then $q$ denotes the adjoint index; that is, the number $p(p-1)^{-1}$. Note that $T$ is a positive $L_{p}$ operator if and only if $T^{*}$ is a positive $L_{q}$ operator. In this case, the definition of the adjoint operator agrees with the usual definition in the Banach space sense.

The following theorem is a standard result. Under the hypothesis one easily shows that the operator is a contraction of both $L_{1}$ and $L_{\infty}$. The conclusion then follows by the Riesz convexity theorem. 
(2.8) Theorem. Let $T$ be a positive operator such that $T \mathbf{1} \leq \mathbf{1}$ and $T^{*} \mathbf{1} \leq \mathbf{1}$. Then $T$ is a positive $L_{p}$ contraction for all $p, 1 \leq p \leq \infty$.

(2.9) Definition. If $T$ is a positive $L_{p}$ operator and $u \in L_{p}$ is a function satisfying $\|T u\|_{p}=\|T\|_{p}\|u\|_{p}$, we say that $u$ is a norming function for $T$. We say that $u$ is semi-invariant for $T$ if $\|T u\|_{p}=\|u\|_{p}$ and both $u$ and $T u$ are strictly positive a.e. A semi-invariant function for a contraction is clearly a norming function.

(2.10) Lemma. If $u$ is a norming function for a positive $L_{p}$ operator $T$, then

$$
T^{*}(T u)^{p-1}=\|T\|_{p}^{p} u^{p-1}
$$

Consequently, if $u$ is semi-invariant for a positive contraction $T$, then $(T u)^{p-1}$ is semi-invariant for $T^{*}$.

Proof.

$$
\begin{aligned}
\|T u\|_{p}^{p} & =\int(T u)(T u)^{p-1} d \nu=\int u T^{*}(T u)^{p-1} d \mu \\
& \leq\|u\|_{p}\left\|T^{*}(T u)^{p-1}\right\|_{q} \leq\|u\|_{p}\left\|T^{*}\right\|_{q}\left\|(T u)^{p-1}\right\|_{q} \\
& =\|u\|_{p}\|T\|_{p}\|T u\|_{p}^{p-1}=\|T u\|_{p}^{p},
\end{aligned}
$$

where the first inequality follows from Hölder's inequality. Thus, we have equality in Hölder's inequality, and so $T^{*}(T u)^{p-1}$ is a constant multiple of $u^{p-1}$.

(2.11) Definition. Suppose $T$ is a positive operator on $\overline{\mathscr{M}}^{+}(d \mu)$. A set $E \in \mathscr{F}$ is called a reducing set for $T$ if $T\left(\chi_{E}\right) \cdot T\left(1-\chi_{E}\right)=0$, where $\chi_{E}$ is the characteristic function of the set $E$.

(2.12) Lemma. The support of a norming function is a reducing set.

Proof. Let $u$ be a norming function for $T$, and $E$ be the support of $u$. Then

$$
\begin{aligned}
\int(T u)^{p-1} T\left(\mathbf{1}-\chi_{E}\right) d \nu & =\int T^{*}(T u)^{p-1}\left(\mathbf{1}-\chi_{E}\right) d \mu \\
& =\|T\|_{p}^{p} \int u^{p-1}\left(\mathbf{1}-\chi_{E}\right) d \mu=0 .
\end{aligned}
$$

Hence $(T u)^{p-1} T\left(\mathbf{1}-\chi_{E}\right)=0$, and so $(T u) T\left(1-\chi_{E}\right)=0$. Now approximate $\frac{1}{u} \chi_{E}$ from below by simple functions. Conclude by $(2.3)$ and positivity that $T\left(\chi_{E}\right) T\left(1-\chi_{E}\right)=0$.

The following lemma concerning functions of a real variable is needed. Observe that the conclusion of the lemma remains valid if we replace $t^{r}$ in the hypothesis by any differentiable function which is strictly monotone almost everywhere.

(2.13) Lemma. Let $\phi, \theta: \mathbb{R}^{+} \rightarrow \mathbb{R}^{+}$be measurable functions satisfying

$$
\begin{aligned}
\int_{0}^{\infty} \phi(t) d t & =\int_{0}^{\infty} \theta(t) d t<\infty, \\
\int_{0}^{\alpha} \phi(t) d t & \leq \int_{0}^{\alpha} \theta(t) d t,
\end{aligned}
$$


and

$$
\int_{0}^{\infty} t^{r} \phi(t) d t=\int_{0}^{\infty} t^{r} \theta(t) d t
$$

for every $\alpha \geq 0$ and some $r>0$. Then $\phi=\theta$ a.e.

Proof.

$$
\begin{aligned}
\int_{0}^{\infty} t^{r} \phi(t) d t & =\int_{0}^{\infty} r s^{r-1}\left(\int_{s}^{\infty} \phi(t) d t\right) d s \\
& \geq \int_{0}^{\infty} r s^{r-1}\left(\int_{s}^{\infty} \theta(t) d t\right) d s=\int_{0}^{\infty} t^{r} \theta(t) d t
\end{aligned}
$$

By (2.15), we have equality. Thus, the set of points at which inequality (2.14) is strict has measure zero. Since

$$
\int_{0}^{\alpha} \phi(t) d t=\int_{0}^{\alpha} \theta(t) d t
$$

for a.a. $\alpha$, and $\phi$ and $\theta$ are positive functions, it follows that $\phi=\theta$ a.e., as desired.

(2.16) Definition. A point transformation $\tau: X \rightarrow X$ is called an automorphism if it is invertible and both $\tau$ and $\tau^{-1}$ are measurable and nonsingular. An automorphism induces two measures, $\mu \circ \tau^{-1}$ and $\mu \circ \tau$, both absolutely continuous with respect to $\mu$. Let $\rho$ denote the Radon-Nikodým derivative of $\mu \circ \tau^{-1}$ with respect to $\mu$. If $1 \leq p<\infty$, then define $Q: L_{p} \rightarrow L_{p}$ by

$$
Q f=\rho^{1 / p}\left(f \circ \tau^{-1}\right)
$$

for $f \in L_{p}$. We call $Q$ the $L_{p}$ isometry induced by $\tau$.

(2.17) Lemma. If $Q$ is the $L_{p}$ isometry induced by an automorphism $\tau$, then $Q^{-1}$ is the $L_{p}$ isometry induced by $\tau^{-1}$ and $Q^{*}$ is the $L_{q}$-isometry induced by $\tau^{-1}$.

Proof. This follows immediately from the definitions if one observes that when $\rho$ is the Radon-Nikodým derivatives of $\mu \circ \tau^{-1}$ with respect to $\mu$, then the Radon-Nikodým derivatives of $\mu \circ \tau$ with respect to $\mu$ is $1 /(\rho \circ \tau)$.

(2.18) Definition. Suppose $1 \leq p<\infty$ and $1 \leq r<\infty$. Define $\psi_{p, r}: L_{p} \rightarrow L_{r}$ by means of the equation

$$
\left[\psi_{p, r}(f)\right](x)=\operatorname{sign}(f(x))|f(x)|^{p / r},
$$

where $\operatorname{sign}(z)$ is the complex number of unit modulus having the same argument as $z$. When $p$ and $r$ are understood, we refer to this embedding simply as $\psi$. Usually $f^{*}$ is used to represent $\psi_{p, q} f$. Perhaps the most important property of $\psi_{p, r}$ is that when $f \in L_{p}$, then $\left\|\psi_{p, r} f\right\|_{r}=\|f\|_{p}^{p / r}$. 
(2.19) Lemma. Let $1 \leq p<\infty$ and $1 \leq r<\infty$. Suppose $Q_{p}$ and $Q_{r}$ are, respectively, the $L_{p}$ and $L_{r}$ isometries induced by an automorphism $\tau$. If $\psi=\psi_{p, r}$ and $f \in L_{p}$, then

$$
Q_{r} \psi f=\psi Q_{p} f
$$

Proof.

$$
\begin{aligned}
Q_{r} \psi f & =\rho^{1 / r}\left[\operatorname{sign}(f)|f|^{p / r}\right] \circ \tau^{-1} \\
& =\operatorname{sign}\left(f \circ \tau^{-1}\right) \rho^{1 / r}\left|f \circ \tau^{-1}\right|^{p / r} \\
& =\operatorname{sign}\left[\rho^{1 / p}\left(f \circ \tau^{-1}\right)\right]\left|\rho^{1 / p}\left(f \circ \tau^{-1}\right)\right|^{p / r} \\
& =\psi Q_{p} f .
\end{aligned}
$$

(2.20) Definition. When $(X, \mathscr{F}, \mu)$ is a measure space and $\mathscr{F}^{\prime}$ is a sub- $\sigma$ algebra of $\mathscr{F}$, then $E\left(\cdot \mid \mathscr{F}^{\prime}\right)$ denotes the conditional expectation operator with respect to $\mathscr{F}^{\prime}$. We adopt the convention that $E\left(f \mid \mathscr{F}^{\prime}\right)$ is 0 on any atom of $\mathscr{F}^{\prime}$ of infinite measure.

(2.21) Theorem (Martingale convergence theorem for finite $\sigma$-algebras). Let $(X, \mathscr{F}, \mu)$ be a $\sigma$-finite measure space. For each $k \geq 1$, suppose $\mathscr{G}_{k}$ is a finite sub- $\sigma$-algebra of $\mathscr{F}$ and $\mathscr{G}_{k} \subseteq \mathscr{G}_{k+1}$. Let $\mathscr{G}_{\infty}=\sigma\left(\bigcup_{k=1}^{\infty} \mathscr{G}_{k}\right)$, the smallest $\sigma$ algebra containing the algebra $\bigcup_{k=1}^{\infty} \mathscr{G}_{k}$. Suppose $1 \leq p<\infty$ and $f \in L_{p}(d \mu)$. Let $f_{k}=E\left(f \mid \mathscr{G}_{k}\right)$ for $1 \leq k \leq \infty$. Then $f_{k} \rightarrow k$ a.e. and in $L_{p}$ norm.

If $p>1$, then the $f_{k}$ 's have a maximal function; more precisely, there is a function $g \in L_{p}^{+}$with $\left|f_{k}\right| \leq g$ for every $k \geq 1$, and $\|g\|_{p} \leq q\|f\|_{p}$.

Proof. See any reference on martingales, e.g. [S, pp. 89-94].

(2.22) Lemma. Let $\left\langle\mathscr{G}_{k}\right\rangle_{k=1}^{\infty}$ be as in the previous theorem and suppose $\left\langle\mathscr{H}_{k}\right\rangle_{k=1}^{\infty}$ is another monotone sequence of finite sub- $\sigma$-algebras of $\mathscr{F}$. Let

$$
\mathscr{H}_{\infty}=\sigma\left(\bigcup_{k=1}^{\infty} \mathscr{H}_{k}\right) .
$$

Let $f \in L_{p}^{+}(d \mu)$, where $1<p<\infty$, and $f_{k}=E\left(f \mid \mathscr{G}_{k}\right)$. Then

$$
E\left(f_{k}^{p} \mid \mathscr{H}_{k}\right) \rightarrow E\left(f_{\infty}^{p} \mid \mathscr{H}_{\infty}\right)
$$

a.e. and in $L_{1}$ norm.

Proof. Let $\phi_{k}=f_{k}^{p}$ for each $k \geq 1$. Then $g=\sup f_{k} \in L_{p}$ by the martingale convergence theorem. Thus $0 \leq \phi_{k} \leq \theta=g^{p} \in L_{1}$, and $\phi_{k} \rightarrow \phi_{\infty}$ a.e. The proof is then completed by the following more general lemma.

(2.23) Lemma. Let $0 \leq \phi_{k} \leq \theta \in L_{1}$ for $k \geq 1$, and let $\phi_{k} \rightarrow \phi_{\infty}$ a.e. Then $E\left(\phi_{k} \mid \mathscr{G}_{k}\right) \rightarrow E\left(\phi_{\infty} \mid \mathscr{G}_{\infty}\right)$ a.e. and in $L_{1}$ norm.

Proof. Let

$$
\xi_{k}=\inf _{n \geq k} \phi_{n} \quad \text { and } \quad \eta_{k}=\sup _{n \geq k} \phi_{n}
$$


Then $\left(\eta_{k}-\xi_{k}\right) \downarrow 0$ a.e. and in $L_{1}$ norm, by the dominated convergence theorem. We have, for any $n \geq k$,

$$
\begin{aligned}
E\left(\xi_{k} \mid \mathscr{G}_{n}\right) & \leq E\left(\xi_{n} \mid \mathscr{G}_{n}\right) \leq E\left(\phi_{n} \mid \mathscr{G}_{n}\right) \\
& \leq E\left(\eta_{n} \mid \mathscr{G}_{n}\right) \leq E\left(\eta_{k} \mid \mathscr{G}_{n}\right) .
\end{aligned}
$$

If $n \rightarrow \infty$ with $k$ fixed, then

$$
E\left(\xi_{k} \mid \mathscr{G}_{\infty}\right) \leq \underline{\lim } E\left(\phi_{n} \mid \mathscr{G}_{n}\right) \leq \varlimsup \lim E\left(\phi_{n} \mid \mathscr{G}_{n}\right) \leq E\left(\eta_{k} \mid \mathscr{G}_{\infty}\right) .
$$

Thus

$$
\begin{aligned}
& \left\|\varlimsup \overline{\lim } E\left(\phi_{n} \mid \mathscr{G}_{n}\right)-\underline{\lim } E\left(\phi_{n} \mid \mathscr{G}_{n}\right)\right\|_{1} \\
& \quad \leq\left\|E\left(\eta_{k} \mid \mathscr{G}_{\infty}\right)-E\left(\xi_{k} \mid \mathscr{G}_{\infty}\right)\right\|_{1} \leq\left\|\eta_{k}-\xi_{k}\right\|_{1}
\end{aligned}
$$

which can be made arbitrarily small. This completes the proof.

We will need the following four lemmas from [AS2], where they are numbered (2.2), (2.3), (2.5), and (2.8) respectively. $L_{p}$ always refers to the case $1<p<$ $\infty$ over a $\sigma$-finite measure space.

(2.24) Lemma. Let $f_{k} \in L_{p}$ for every $k, 1 \leq k \leq n$. If $V: L_{p} \rightarrow L_{p}$ is a positive bounded linear operator, then

$$
\max _{1 \leq k \leq n}\left|V f_{k}\right| \leq V\left(\max _{1 \leq k \leq n}\left|f_{k}\right|\right)
$$

and, consequently,

$$
\left\|\max _{1 \leq k \leq n}\left|V f_{k}\right|\right\|_{p} \leq\|V\| \cdot\left\|\max _{1 \leq k \leq n}\left|f_{k}\right|\right\|_{p} .
$$

(2.25) Lemma. For each $\varepsilon>0$ there is a $\delta>0$ such that if $E: L_{p} \rightarrow L_{p}$ is a conditional expectation operator, $f \in L_{p}$, and $\|f\|_{p}-\|E f\|_{p}<\delta\|f\|_{p}$, then $\|f-E f\|_{p}<\varepsilon\|f\|_{p}$.

(2.26) Lemma. Let $f_{k m} \in L_{p}$ for every $m \geq 0$ and every $k, 1 \leq k \leq n$. If $\lim _{m \geq 0}\left\|f_{k m}-f_{m}\right\|_{p}=0$ for each $k$, then

$$
\lim _{m \geq 0}\left\|\max _{1 \leq k \leq n}\left|f_{k m}\right|-\max _{1 \leq k \leq n}\left|f_{k}\right|\right\|_{p}=0 .
$$

(2.27) Lemma. Let $\left\langle f_{n}\right\rangle_{n=0}^{\infty}$ be a sequence of functions in $L_{p}$ such that $\left(\sup _{n \geq 0}\left|f_{n}\right|\right) \in L_{p}$. Then $\left\langle f_{n}\right\rangle_{n=0}^{\infty}$ converges a.e. if and only if

$$
\lim _{n \geq 0}\left\|\sup _{k \geq n}\left|f_{k}-f_{n}\right|\right\|_{p}=0 .
$$

The following are analogous to Lemmas (2.6) and (2.7) in [AS2]. The first one follows from a result of Mazur [M], since the mapping $\psi_{p, r}$ may be regarded as a composition of his map $F$ from $L_{1}$ to $L_{r}$ and his map $G$ from $L_{p}$ to $L_{1}$, both uniformly continuous on the unit ball. 
(2.28) Lemma (Uniform continuity of $\psi_{p, r}$ ). Let $1 \leq p<\infty$ and $1 \leq r<\infty$. Given $\varepsilon>0$ and $M>0$, there is $a \delta>0$ depending only on $\varepsilon, M, p$, and $r$ such that $\|\psi f-\psi g\|_{r}<\varepsilon$ whenever $\|f\|_{p} \leq M,\|g\|_{p} \leq M$, and $\|f-g\|_{p}<\delta$. (2.29) Lemma. Given $\varepsilon>0$ and $M>0$ there is $a \delta>0$ depending only on $\varepsilon$, $M, p$, and $r$ such that if $\left\langle f_{k}\right\rangle_{k=0}^{\infty}$ is a sequence in $L_{p}$ with $\left\|\sup _{k \geq 0}\left|f_{k}\right|\right\|_{p} \leq M$ and $\left\|\sup _{k \geq 0}\left|f_{k}-f_{0}\right|\right\|_{p}<\delta$ then

$$
\left\|\sup _{k \geq 0}\left|\psi f_{k}-\psi f_{0}\right|\right\|_{r}<\varepsilon .
$$

Proof. Let $\delta$ be as given in the uniform continuity of $\psi$ corresponding to $\varepsilon / 2$, $M, p$, and $r$. Let $n \geq 1$ be given. Fix a partition $\left\{A_{1}, \ldots, A_{n}\right\}$ of $X$ such that

$$
\max _{0 \leq k \leq n}\left|\psi f_{k}-\psi f_{0}\right|=\sum_{m=1}^{n}\left|\psi f_{m}-\psi f_{0}\right| \chi_{A_{m}} .
$$

Let $f=\sum_{m=1}^{n} f_{m} \chi_{A_{m}}$, so that

$$
\max _{0 \leq k \leq n}\left|\psi f_{k}-\psi f_{0}\right|=\left|\psi f-\psi f_{0}\right| \text {. }
$$

We have $\|f\|_{p} \leq M,\left\|f_{0}\right\|_{p} \leq M$, and $\left\|f-f_{0}\right\| \leq\left\|\sup _{k \geq 0}\left|f_{k}-f_{0}\right|\right\|_{p}$. Therefore, if this last norm is less than $\delta$, the uniform continuity of $\psi$ implies that $\left\|\psi f-\psi f_{0}\right\|_{r}<\varepsilon / 2$. This completes the proof.

We also need the following, which is an immediate consequence of

$$
\left\|T_{n} f_{n}-T f\right\|_{p} \leq\left\|T_{n}\right\| \cdot\left\|f_{n}-f\right\|_{p}+\left\|T_{n} f-T f\right\|_{p} .
$$

(2.30) Lemma. Suppose $\left\langle T_{n}\right\rangle_{n=1}^{\infty}$ and $T$ are $L_{p}$ contractions and

$$
\lim _{n \geq 1}\left\|T_{n} f-T f\right\|_{p}=0
$$

whenever $f \in L_{p}$. If $f_{n} \rightarrow f$ in $L_{p}$ norm, then

$$
\lim _{n \geq 1}\left\|T_{n} f_{n}-T f\right\|_{p}=0 \text {. }
$$

\section{INDUCED OPERATORS}

In this section, we will be interested primarily in positive $L_{p}$ operators with strictly positive norming functions. We begin, however, with two more general lemmas.

(3.1) Lemma. Let $T$ be a positive operator on $\overline{\mathscr{M}}^{+}(d \mu)$. Suppose $u \in \mathscr{M}^{+}(d \mu)$ is strictly positive. If there is a $\lambda \in \mathbb{R}^{+}$such that

$$
T^{*}(T u)^{p-1} \leq \lambda^{p} u^{p-1},
$$

then $T$ is a positive $L_{p}$ operator with $\|T\|_{p} \leq \lambda$.

(3.3) Remarks. In the Borel case, this follows from a result in [AS1] concerning dilations. The general case was considered in [K1]. We have included the following short proof to make this paper more self-contained. 
Proof. If $\lambda=0$, it is easy to see that $T=0$, since $\int(T u)^{p-1}(T f) d \mu=0$ for every $f \in \overline{\mathscr{M}}^{+}(d \mu)$.

Suppose $\lambda>0$ and let $v=T u$. Because of (3.2), the $\sigma$-finiteness of $\mu$ and the fact that $u$ is finite a.e., one argues that $v$ is finite a.e. (The proof is essentially contained in [AS1, p. 391].)

Let $d \mu^{\prime}=u^{p} d \mu$ and $d \nu^{\prime}=(v / \lambda)^{p} d \nu$. Define an operator $R: \overline{\mathscr{M}}^{+}\left(d \mu^{\prime}\right) \rightarrow$ $\overline{\mathscr{M}}^{+}\left(d \nu^{\prime}\right)$ by $R f=\chi_{G} \frac{1}{v} T(u f)$ for $f \in \overline{\mathscr{M}}^{+}\left(d \mu^{\prime}\right)$, where $G$ is the support of $v$. This is clearly a positive operator in the sense of (2.4). A routine computation shows that the adjoint, $R^{*}: \overline{\mathscr{M}}^{+}\left(d \nu^{\prime}\right) \rightarrow \overline{\mathscr{M}}^{+}\left(d \mu^{\prime}\right)$, is given by

$$
R^{*} g=\frac{1}{\lambda^{p} u^{p-1}} T^{*}\left(v^{p-1} g\right)
$$

for $g \in \overline{\mathscr{M}}^{+}\left(d \nu^{\prime}\right)$. Thus $R 1 \leq 1$ and $R^{*} 1 \leq 1$, so by Theorem (2.8), $R$ is an $L_{p}$ contraction. This means that if $f \in \overline{\mathscr{M}}^{+}\left(d \mu^{\prime}\right)$, then

$$
\int(R f)^{p} d \nu^{\prime} \leq \int f^{p} d \mu^{\prime}
$$

If $f \in \overline{\mathscr{M}}^{+}(d \mu)$, then $f=u \tilde{f}$ for some $\tilde{f} \in \overline{\mathscr{M}}^{+}\left(d \mu^{\prime}\right)$. Hence

$$
\begin{aligned}
\int(T f)^{p} d \nu & =\int[T(u \tilde{f})]^{p} d \nu=\lambda^{p} \int(R \tilde{f})^{p} d \nu^{\prime} \\
& \leq \lambda^{p} \int \tilde{f}^{p} d \mu^{\prime}=\lambda^{p} \int f^{p} d \mu .
\end{aligned}
$$

This shows that $T$ is an $L_{p}$ operator with $\|T\|_{p} \leq \lambda$.

(3.4) Lemma. Let $T$ be a positive operator on $\overline{\mathscr{M}}^{+}(d \mu)$. Suppose $u \in \mathscr{M}^{+}(d \mu)$ is strictly positive, and that there is a $\lambda \in \mathbb{R}^{+}$such that

$$
T^{*}(T u)^{p-1} \leq \lambda^{p} u^{p-1} \text {. }
$$

Let $v=T u$ and let $G$ be the support of $v$. Let $r$ be any exponent, $1<r<\infty$. Then

$$
S f=\chi_{G}\left(\frac{v}{\lambda}\right)^{p / r-1} T\left(u^{1-p / r} f\right),
$$

for $f \in \overline{\mathscr{M}}^{+}(d \mu)$, defines a positive $L_{r}$ operator $S: \overline{\mathscr{M}}^{+}(d \mu) \rightarrow \overline{\mathscr{M}}^{+}(d \nu)$ with $\|S\|_{r} \leq \lambda$.

Proof. $S^{*}: \overline{\mathscr{M}}^{+}(d \nu) \rightarrow \overline{\mathscr{M}}^{+}(d \mu)$ is easily calculated; one sees that for $g \in$ $\overline{\mathscr{M}}^{+}(d \nu)$,

$$
S^{*} g=(\lambda u)^{1-p / r} T^{*}\left(v^{p / r-1} \chi_{G} g\right) .
$$

Let $\tilde{u}=u^{p / r}$. Then $\tilde{u}$ is strictly positive a.e., and $S^{*}(S u)^{r-1} \leq \lambda^{r} \tilde{u}^{r-1}$. Thus, Lemma (3.1) completes the proof.

(3.5) Lemma. Suppose $u_{1}$ and $u_{2}$ are strictly positive norming functions for $a$ positive $L_{p}$ operator $T$ on $\overline{\mathscr{M}}^{+}(d \mu)$. For any $\alpha \in \mathbb{R}^{+}$, the set

is a reducing set for $T$.

$$
E_{\alpha}=\left\{x \in X \mid \frac{u_{2}(x)}{u_{1}(x)}>\alpha\right\}
$$


Proof. As in the proof of Lemma (3.1), let $d \mu^{\prime}=u_{1}^{p} d \mu$ and $d \nu^{\prime}=\left(v_{1} / \lambda\right)^{p} d \nu$, where $v_{1}=T u_{1}$ and $\lambda=\|T\|_{p}$. Observe that even if $v_{1}$ is not strictly positive a.e., its support is equal to the support of $v_{2}=T u_{2}$ a.e. Without loss of generality then, we may replace the set $Y$ with this common support. Define $R: \overline{\mathscr{M}}^{+}\left(d \mu^{\prime}\right) \rightarrow \overline{\mathscr{M}}^{+}\left(d \nu^{\prime}\right)$ for $f \in \overline{\mathscr{M}}^{+}\left(d \mu^{\prime}\right)$ by $R f=T\left(u_{1} f\right) / v_{1}$.

$R \mathbf{1}=R^{*} \mathbf{1}=\mathbf{1}$, so $R$ is an $L_{p}$ contraction. 1 is a norming function for $R$; we now show that $u=u_{2} / u_{1}$ is another. One may verify that $R^{*}(R u)^{p-1}=$ $u^{p-1}$, from which $\|R u\|_{p}=\|u\|_{p}$ easily follows. Let $v=R u$.

Let $\alpha \geq 0$ be arbitrary. Let $u_{\alpha}=u \wedge \alpha$, the function $u$ truncated at the value $\alpha$. Observe that $E_{\alpha}$ is the support of $u-u_{\alpha}$. Also note that $R u_{\alpha} \leq v_{\alpha}=v \wedge \alpha$, hence

$$
\int u_{\alpha} d \mu^{\prime}=\int R u_{\alpha} d \nu^{\prime} \leq \int v_{\alpha} d \nu^{\prime} .
$$

Let $\phi: \mathbb{R}^{+} \rightarrow \mathbb{R}^{+}$be the distribution of $u$; that is, $\phi(t)=\mu^{\prime}\{x: u(x) \geq t\}$. Let $\theta$ be the distribution of $v$, similarly defined with respect to $\nu^{\prime}$. Inequality (3.6) has the equivalent form

$$
\int_{0}^{\alpha} \phi(t) d t \leq \int_{0}^{\alpha} \theta(t) d t
$$

Since $\|u\|_{p}=\|v\|_{p}$, we have

$$
\int_{0}^{\infty} t^{p-1} \phi(t) d t=\int_{0}^{\infty} t^{p-1} \theta(t) d t
$$

Finally, $u \in L_{1}\left(d \mu^{\prime}\right)$, since $p>1$ and $\mu^{\prime}$ is a finite measure. Since $\|u\|_{1}=$ $\|v\|_{1}$, we have

$$
\int_{0}^{\infty} \phi(t) d t=\int_{0}^{\infty} \theta(t) d t<\infty .
$$

Conditions (3.7)-(3.9) allow us to invoke Lemma (2.13) and conclude that $\phi=\theta$ a.e. in Lebesgue measure. Since

$$
\left\|u-u_{\alpha}\right\|_{p}^{p}=p \int_{\alpha}^{\infty} t^{p-1} \phi(t) d t,
$$

we have

$$
\left\|u-u_{\alpha}\right\|_{p}=\left\|v-v_{\alpha}\right\|_{p} \leq\left\|R\left(u-u_{\alpha}\right)\right\|_{p},
$$

where the inequality follows because $R u_{\alpha} \leq v_{\alpha}$. As $R$ is a contraction, we conclude that the norms are in fact equal. Thus, $u-u_{\alpha}$ is a norming function. By Lemma (2.12), then, its support is a reducing set for $R$. It easily follows that $E_{\alpha}$ also reduces $T$.

(3.10) Remarks. One may replace the "less than" in the definition of $E_{\alpha}$ by any other inequality, simply by considering complements or reversing the roles of $u_{1}$ and $u_{2}$. The complement of a reducing set is a reducing set; it is also easy 
to show that the intersection of reducing sets is a reducing set. In fact, the class of reducing sets of a bounded $L_{p}$ operator is a sub- $\sigma$-algebra of the underlying measure space. This is shown in [K2], which also includes a different proof of the above lemma.

(3.11) Theorem. Suppose $T$ is a positive $L_{p}$ operator on $\overline{\mathscr{M}^{+}}(d \mu)$, and $u_{1}$ and $u_{2}$ are strictly positive norming functions for $T$. Let $v_{i}=T u_{i}$ for $i=1,2$ and let $G$ be the support of the $v_{i}$ 's. Let $1<r<\infty$, and define positive operators $S_{1}$ and $S_{2}$ on $\overline{\mathscr{M}}^{+}(d \mu)$ by

$$
S_{i} f=\chi_{G}\|T\|_{p}^{1-p / r} v_{i}^{p / r-1} T\left(u_{i}^{1-p / r} f\right)
$$

for $f \in \overline{\mathscr{M}}^{+}(d \mu)$ and $i=1,2$. Then $S_{1} f=S_{2} f$ a.e. for every $f \in \overline{\mathscr{M}}^{+}(d \mu)$. Proof. By (2.3), it suffices to consider $f \in \mathscr{M}^{+}(d \mu)$.

Let $s=p / r-1$. If $s=0$, there is nothing to prove. Otherwise, let $\varepsilon>0$ be given, and choose a positive integer $N>1 / \varepsilon$.

For each $n \geq 1$, let

$$
E_{n}=\left\{x \in X \mid \frac{N+n-1}{N}<\frac{u_{2}(x)}{u_{1}(x)} \leq \frac{N+n}{N}\right\}
$$

and

$$
E_{-n}=\left\{x \in X \mid \frac{N+n-1}{N}<\frac{u_{1}(x)}{u_{2}(x)} \leq \frac{N+n}{N}\right\} .
$$

Also, let $E_{0}$ be the set of points in $A$ where $u_{1}(x)=u_{2}(x)$. Then $\left\{E_{n} \mid n \in \mathbb{Z}\right\}$ is a partition of $X$ into reducing sets.

Let $f \in \overline{\mathscr{M}}^{+}(d \mu)$ be given and let $f_{n}=f \chi_{E_{n}}$ for every $n \in \mathbb{Z}$. The $f_{n}$ 's have disjoint support, as do the functions $T\left(u_{1}^{-s} f_{n}\right)$ and $T\left(u_{2}^{-s} f_{n}\right)$.

Now suppose $n \geq 1$ and $s>0$. Since $T$ is positive, we have

$$
\left(\frac{N}{N+n}\right)^{s} T\left(\frac{f_{n}}{u_{1}^{s}}\right) \leq T\left(\frac{f_{n}}{u_{2}^{s}}\right) \leq\left(\frac{N}{N+n-1}\right)^{s} T\left(\frac{f_{n}}{u_{1}^{s}}\right) .
$$

Let $u_{i n}=u_{i} \chi_{E_{n}}$ and $v_{i n}=T\left(u_{i n}\right)$ for every $n \in \mathbb{Z}$ and $i=1,2$. The functions $T\left(u_{i}^{-s} f_{n}\right)$ and $v_{m i}$ will have disjoint supports unless $m=n$; thus $S_{i} f_{n}$ depends only on $T\left(u_{i}^{-s} f_{n}\right)$ and $v_{n i}^{s}$. We have

$$
\left(\frac{N+n-1}{N}\right)^{s} v_{n 1}^{s} \leq v_{n 2}^{s} \leq\left(\frac{N+n}{N}\right)^{s} v_{n 1}^{s} \text {. }
$$

Therefore,

$$
\left(\frac{N+n-1}{N+n}\right)^{s} S_{1} f_{n} \leq S_{2} f_{n} \leq\left(\frac{N+n}{N+n-1}\right)^{s} S_{1} F_{N}
$$

If $\left(S_{1} f_{n}\right)(x)=0$, then $\left(S_{2} f_{n}\right)(x)$ must be zero as well. Otherwise,

$$
\left|\left(\frac{\left(S_{2} f_{n}\right)(x)}{\left(S_{1} f_{n}\right)(x)}\right)^{1 / s}-1\right| \leq \frac{1}{N+n-1}<\varepsilon .
$$


If $s<0$, then the order of the terms in (3.14) is reversed, but (3.15) remains valid.

If $n \leq-1$, the argument is symmetric, with the conclusion

$$
\left|\left(\frac{\left(S_{1} f_{n}\right)(x)}{\left(S_{2} f_{n}\right)(x)}\right)^{1 / s}-1\right| \leq \frac{1}{N+n-1}<\varepsilon .
$$

It is clear that $S_{1} f_{0}=S_{2} f_{0}$. Since $\varepsilon>0$ is arbitrary, we conclude that $S_{1} f_{n}=S_{2} f_{n}$ a.e. for each $n \in \mathbb{Z}$. Thus $S_{1} f=S_{2} f$ a.e., as desired.

(3.16) Theorem. Suppose $1<p<\infty$ and $1<r<\infty$. Let $T$ be a positive $L_{p}$ operator with a strictly positive norming function $u$. Let $v=T u$ and let $G$ be the support of $v$. Then

$$
T_{r} f=\chi_{g}\|T\|^{1-p / r} v^{p / r-1} T\left(u^{1-p / r} f\right),
$$

for $f \in \overline{\mathscr{M}}^{+}(d \mu)$, defines a positive $L_{r}$ operator $T_{r}: \overline{\mathscr{M}}^{+}(d \mu) \rightarrow \overline{\mathscr{M}}^{+}(d \nu)$ such that $\left\|T_{r}\right\|_{r}=\|T\|_{p}$. This operator, called the $L_{r}$ operator induced by $T$, is independent of the choice of $u$.

Proof. Whether $T$ is given as an $L_{p}$ operator in the Banach space sense or in the sense of Definition (2.4), it is clear that $T_{r}$ is a positive operator in the sense of (2.4). Lemmas (2.10) and (3.4) combine to show that $T_{r}$ is in fact an $L_{r}$ operator with norm bounded by $\|T\|_{p}$. To see that this norm is actually achieved, let $f=u^{p / r}$. Theorem (3.11) demonstrates that $T_{r}$ does not depend on the choice of norming function.

(3.17) Corollary. Suppose $T$ is an $L_{p}$ contraction with a semi-invariant function where $1<p<\infty$. For every $r, 1<r<\infty$,

$$
T_{r} f=v^{p / r-1} T\left(u^{1-p / r} f\right)
$$

defines a positive contraction of $L_{r}$.

(3.18) Remarks. If $T$ is an $L_{p}$ isometry induced by an automorphism $\tau$ (as in (2.16)), then $T_{r}$ is simply the $L_{r}$ isometry induced by $\tau$. When the underlying space has finite measure, we may take $u=1$ and $v=\rho^{1 / p}$. The general $\sigma$-finite case is not much harder to check.

A larger and more important class of operators has the form $E Q E$, where $Q$ is an $L_{p}$ isometry induced by an automorphism and $E$ is a conditional expectation operator of finite rank. Such operators where crucial to the proof of the pointwise ergodic theorem for positive $L_{p}$ contractions (see [A]). Thus, the following lemma is of some general interest as well as being necessary for $\S 5$ of this paper.

(3.19) Lemma. Suppose $1<p<\infty, 1<r<\infty$, and that $Q_{p}$ and $Q_{r}$ are, respectively, the $L_{p}$ and $L_{r}$ isometries induced by an automorphism $\tau$ over a measure space $(X, \mathscr{F}, \mu)$. Let $\widetilde{F}$ be a sub- $\sigma$-algebra of $\mathscr{F}$ and let $\tilde{\mu}$ be the 
restriction of $\mu$ to $\widetilde{F}$. Let $E$ be conditional expectation with respect to $\widetilde{F}$ and suppose

$$
T: L_{p}(X, \widetilde{F}, \tilde{\mu}) \rightarrow L_{p}(X, \widetilde{F}, \tilde{\mu})
$$

is given by $T=E Q_{p} E$. If $T$ has a semi-invariant function $u$, then $T_{r}=E Q_{r} E$.

Proof. Let $v=T u$. For $f \in L_{r}(X, \widetilde{F}, \tilde{\mu})$, we have

$$
\begin{aligned}
T_{r} f & =v^{p / r-1} T\left(u^{1-p / r} f\right) \\
& =v^{p / r-1} E\left(\rho^{1 / p}\left(u \circ \tau^{-1}\right)^{1-p / r}\left[(E f) \circ \tau^{-1}\right]\right) \\
& =v^{p / r-1} E\left[\rho^{1 / p}\left(u \circ \tau^{-1}\right)^{1-p / r}\left(f \circ \tau^{-1}\right)\right],
\end{aligned}
$$

where the third line follows because $f$ is already $\widetilde{F}$-measurable. Because $\|v\|_{p}=\|u\|_{p}, Q$ is an isometry and $p>1$, we conclude that $Q_{p} u$ must already be $\widetilde{F}$-measurable, lest some norm be lost in taking the conditional expectation. Thus $v=\rho^{1 / p}\left(u \circ \tau^{-1}\right)$ and

$$
\begin{aligned}
T_{r} f & =E\left[v^{p / r-1} \rho^{1 / p}\left(u \circ \tau^{-1}\right)^{1-p / r}\left(f \circ \tau^{-1}\right)\right] \\
& =E\left[\left(\rho^{1 / p}\right)^{p / r-1}\left(u \circ \tau^{-1}\right)^{p / r-1} \rho^{1 / p}\left(u \circ \tau^{-1}\right)^{1-p / r}\left(f \circ \tau^{-1}\right)\right] \\
& =E\left[\rho^{1 / r}\left(f \circ \tau^{-1}\right)\right]=E Q_{r} f=E Q_{r} E f . \quad \square
\end{aligned}
$$

(3.20) Lemma. Let $1<p<\infty, 1<r<\infty$, and let $Q$ be the $L_{p}$ isometry induced by an automorphism $\tau$. Let $T=E Q E$ for some conditional expectation operator $E$. If $T$ has a semi-invariant function and $R=R_{r}$ is the $L_{r}$ isometry induced by $\tau^{-1}$, then $\left(T^{*}\right)_{r}=E R E$.

Proof. $\left(T^{*}\right)_{r}=\left(E Q^{*} E\right)_{r}=\left(E R_{q} E\right)_{r}=E R E$. We have used the self-adjointness of $E$ and Lemmas (3.19) and (2.17) for the fact that $Q^{*}$ is the $L_{r}$ isometry induced by $\tau^{-1}$.

\section{FINITE-DIMENSIONAL APPROXIMATION}

In $[A K]$, it was shown that all positive contractions over the unit interval are induced by a point mapping of some type, followed by a conditional expectation. For positive contractions with semi-invariant functions, the argument is easier and does not require the underlying space to be interval. However, we will want to extract a point mapping from a set mapping, so we will require our measure spaces to be Lebesgue spaces. That is, a measure space $(X, \mathscr{F}, \mu)$ where $X$ is a complete metric space and $\mathscr{F}$ is the Borel $\sigma$-algebra. We allow the space to have $\sigma$-finite measure. Since a separable metric space is second countable, the $\sigma$-algebra of measurable sets in a Lebesgue space can always be generated by a countable algebra of sets.

The details of the construction give us a family of finite-dimensional operators $\left\langle T^{n}\right\rangle_{n=1}^{\infty}$ (these are ordinary superscripts, not powers), each with a semiinvariant function $u_{n}$, where $u_{n} \rightarrow u$ a.e. Furthermore, these operators have 
the property that $\left(T^{n}\right)_{r} f \rightarrow T_{r} f$ a.e. and in $L_{r}$ norm for every $f \in L_{r}$. These finite-dimensional approximations to the induced operator provide the key to the proof of the Theorem (1.2).

(4.1) Definitions. Let $\mathbf{X}=(X, \mathscr{F}, \mu)$ be a $\sigma$-finite Lebesgue space and suppose $T: L_{p}(d \mu) \rightarrow L_{p}(d \mu)$ has a semi-invariant function $u$. Let $\mathbf{I}=(I, \mathscr{B}, m)$ be the usual Lebesgue space of the unit interval. Let $\mathbf{W}=(W, \mathscr{K}, \omega)=\mathbf{X} \times \mathbf{I}$.

Let $\mathscr{I}=\{F \times I \mid F \in \mathscr{F}\}$, the "vertical" sub- $\sigma$-algebra of $\mathscr{K}$, and let $v$ be the $\mathscr{I}$-measurable function given by $v(x, y)=(T u)(x)$ for every $y$ in the unit interval.

Suppose $\left\langle\mathscr{F}_{n}\right\rangle_{n=1}^{\infty}$ is an increasing sequence of finite sub- $\sigma$-algebras of $\mathscr{F}$ such that $\sigma\left(\bigcup_{n=1}^{\infty} \mathscr{F}_{n}\right)=\mathscr{F}$. That is, $\mathscr{F}$ is the smallest $\sigma$-algebra containing all the $\mathscr{F}_{n}$ 's. Let $\mathscr{I}_{n}=\left\{F \times I \mid F \in \mathscr{F}_{n}\right\}$.

For each $n \geq 1$, fix an enumeration $\left\{F_{n, i}\right\}_{i=1}^{k_{n}}$ of the atoms of $\mathscr{F}_{n}$. Let $\gamma_{n, 0}=0$, and for each $i, 1 \leq i \leq k_{n}$, let

$$
\gamma_{n, i}=T\left(u \sum_{j=1}^{i} \chi_{F_{n, j}}\right)
$$

and

$$
H_{n, i}=\left\{(x, y) \in W \mid \frac{\gamma_{n, i-1}(x)}{(T u)(x)}<y \leq \frac{\gamma_{n, i}(x)}{(T u)(x)}\right\} .
$$

Let $\mathscr{H}_{n}$ be the finite sub- $\sigma$-algebra of $\mathscr{K}$ generated by the partition $\left\{H_{n, i}\right\}_{i=1}^{k_{n}}$ of $W$. Let $\Pi_{n}$ be the set mapping from $\mathscr{F}_{n}$ to $\mathscr{H}_{n}$ determined by $\Pi_{n} F_{n, i}=$ $H_{n, i}$ for each $i, 1 \leq i \leq n$.

(4.2) Lemma. There is a point mapping $\pi: W \rightarrow X$ such that $\pi^{-1} F_{n, i}=H_{n, i}$ for every $n \geq 1$ and every $i, 1 \leq i \leq k_{n}$.

Proof. The family of set mappings $\Pi_{n}$ determines a unique set mapping of the algebra $\bigcup_{n=1}^{\infty} \mathscr{F}_{n}$, because of $\mathscr{F}_{n}$ 's form a monotone sequence. This mapping preserves unions and complements, and it extends to a homomorphism of the measure algebras of $(X, \mathscr{F})$ and $(W, \mathscr{K})$. Since the sets underlying both spaces are complete metric spaces, there is a point mapping $\pi$ defined from almost all of $W$ onto almost all of $X$ which induces the set mapping (see [Ry, p. 329]). Thus if $\Pi F=H$, then $\pi^{-1} F=H$. Since $\Pi F_{n, i}=\Pi_{n} F_{n, i}$, the desired result follows.

(4.3) Lemma. For every $F \in \mathscr{F}, \int_{F} u^{p} d \mu=\int_{\pi^{-1} F} v^{p} d \omega$. 
Proof. If $F=F_{n, i} \in \mathscr{F}$ for some $n \geq 1$ and some $i, 1 \leq i \leq k_{n}$, then

$$
\begin{aligned}
\int_{\pi^{-1} F} v^{p} d \omega & =\int_{X}(T u)^{p}\left[\frac{\gamma_{n, i}-\gamma_{n, i-1}}{T u}\right] d \mu \\
& =\int_{X}(T u)^{p-1} T\left(u \chi_{F}\right) d \mu \\
& =\int_{F} u T^{*}(T u)^{p-1} d \mu=\int_{F} u^{p} d \mu .
\end{aligned}
$$

The lemma is true for a generating subalgebra of $\mathscr{F}$. The proof is easily completed.

(4.4) Lemma. Suppose $\phi$ is an $\mathscr{F}$-measurable function and $\theta$ is a $\mathscr{K}$-measurable function with $\phi>0 \quad \mu$-a.e. and $\theta>0$ w-a.e. such that

$$
\int_{F} \phi d \mu=\int_{\pi^{-1} F} \theta d \omega
$$

for every $F \in \mathscr{F}$. Then, if $1 \leq p<\infty$,

$$
S f=\left(\frac{\theta}{\phi \circ \pi}\right)^{1 / p}(f \circ \pi),
$$

for $f \in L_{p}(d \mu)$, defines an isometry $S: L_{p}(d \mu) \rightarrow L_{p}(d \omega)$.

Proof. First suppose $f=\phi^{1 / p} \chi_{F}$ for some $F \in \mathscr{F}$. Then

$$
\begin{aligned}
\|S f\|_{p}^{p} & =\int_{Z} \frac{\theta}{\phi \circ \pi}\left(\phi^{1 / p} \chi_{F}\right)^{p} \circ \pi d \omega \\
& =\int_{\pi^{-1} F} \theta d \omega=\int_{F} \phi d \mu=\|f\|_{p}^{p} .
\end{aligned}
$$

In the general case, approximate $f \phi^{-1 / p}$ by $\mathscr{F}$-simple functions.

This isometry yields a result analogous to the theorem of Akcoglu and Koop [AK].

(4.5) Theorem. Define $Q: L_{p}(d \mu) \rightarrow L_{p}(d \omega)$ by

$$
Q=\frac{v}{u \circ \pi}(f \circ \pi) \text { for } f \in L_{p}(d \mu) .
$$

If $\omega^{\prime}$ is $\omega$ restricted to $\mathscr{I}$, and we identify $\mathbf{X}$ with $\left(W, \mathscr{I}, \omega^{\prime}\right)$, then $T f=$ $E(Q f \mid \mathcal{F})$ for every $F \in L_{p}(d \mu)$.

Proof. By the two previous lemmas, we see that $Q$ is an isometry of the indicated spaces. Suppose $f=u \chi_{F}$ for some $F \in \mathscr{F}$. Then

$$
\begin{aligned}
{[E(Q f \mid \mathcal{F})](x) } & =\int_{0}^{1}(Q f)(x, y) d y=\int_{0}^{1} v(x, y) \chi_{\pi^{-1} F}(x, y) d y \\
& =(T u)(x)\left(\frac{T\left(u \chi_{F}\right)(x)}{(T u)(x)}\right)=(T f)(x) .
\end{aligned}
$$


For a general $\mathscr{F}$-measurable $f$, approximate $f u^{-1}$ by $\mathscr{F}$-simple functions.

(4.6) Lemma. Suppose $\widetilde{F}$ is a finite sub- $\sigma$-algebra of $\mathscr{F}$ and $\tilde{\mu}$ is the restriction of $\mu$ to $\widetilde{\mathscr{F}}$. If $\tilde{v} \in L_{p}(d \omega)$ satisfies $\tilde{v}>0$ a.e., then there is a unique $\tilde{\mathscr{F}}$. measurable function $\tilde{u}$ such that, for every $F \in \widetilde{\mathscr{F}}$,

$$
\int_{F} \tilde{u}^{p} d \mu=\int_{\pi^{-1} F} \tilde{v}^{p} d \omega .
$$

Furthermore, the mapping

$$
f \in L_{p}(X, \widetilde{\mathscr{F}}, \tilde{\mu}) \mapsto \frac{\tilde{v}}{\tilde{u} \circ \pi}(f \circ \pi) \in L_{p}(d \omega)
$$

is an isometry which transforms $\tilde{u}$ to $\tilde{v}$.

Proof. Let $\left\{F_{i}\right\}_{i=1}^{k}$ be an enumeration of the atoms of $\widetilde{\mathscr{F}}$. Let $H_{i}=\pi^{-1} F_{i}$ for each $i, 1 \leq i \leq k$. Then

$$
\tilde{u}=\sum_{i=1}^{k}\left(\frac{1}{\mu F_{i}} \int_{H_{i}} \tilde{v}^{p} d \omega\right)^{1 / p} \chi_{F_{i}} .
$$

If $f=\sum_{i=1}^{k} c_{i} \chi_{F_{i}} \in L_{p}(X, \widetilde{\mathscr{F}}, \tilde{\mu})$, then

$$
\left\|\frac{\tilde{v}}{\tilde{u} \circ \pi}(f \circ \pi)\right\|_{p}^{p}=\sum_{i=1}^{k} c_{i}^{p} \int_{\pi^{-1} F_{i}} \frac{\tilde{v}^{p}}{(\tilde{u} \circ \pi)^{p}} d \omega=\sum_{i=1}^{k} c_{i}^{p} \mu F_{i}=\|f\|_{p}^{p},
$$

as desired.

(4.7) Theorem. For each $n \geq 1$, let $v_{n}=E\left(v \mid \mathscr{F}_{n}\right)$. Let $u_{n}$ be the corresponding $\mathscr{F}_{n}$-measurable functions as given by Lemma (4.6). Then $u_{n} \rightarrow u$ u-a.e.

Proof. Let $u_{n}=\sum_{i=1}^{k_{n}} u_{n, i} \chi_{F_{n, i}}$. Then

$$
\begin{aligned}
u_{n, i}^{p} & =\frac{1}{\mu F_{n, i}} \int_{H_{n, i}} v_{n}^{p} d \omega \\
& =\left(\frac{1}{\mu F_{n, i}} \int_{F_{n, i}} u^{p} d \mu\right) \frac{\left.\left(\omega H_{n, i}\right)^{-1} \int_{H_{n, i}} v_{n}^{p} d \omega\right)}{\left.\left(\omega H_{n, i}\right)^{-1} \int_{H_{n, i}} v^{p} d \omega\right)} .
\end{aligned}
$$

Thus

$$
u_{n}^{p} \circ \pi=\frac{E\left(v_{n}^{p} \mid \mathscr{H}_{n}\right)}{E\left(v^{p} \mid \mathscr{H}_{n}\right)}\left[E\left(u^{p} \mid \mathscr{F}_{n}\right) \circ \pi\right]
$$

By the martingale convergence theorem, with $p=1$, we have $E\left(u^{p} \mid \mathscr{F}_{n}\right) \rightarrow u^{p}$ $\mu$-a.e., and $E\left(v^{p} \mid \mathscr{H}_{n}\right) \rightarrow E\left(v^{p} \mid \mathscr{H}\right) \quad \omega$-a.e. By Lemma (2.22), we also have $E\left(v_{n}^{p} \mid \mathscr{H}_{n}\right) \rightarrow E\left(v^{p} \mid \mathscr{H}\right) \quad \omega$-a.e. Therefore

$$
u_{n}^{p} \circ \pi \rightarrow E\left(u^{p} \mid \mathscr{F}_{n}\right) \circ \pi,
$$

and so $u_{n}^{p} \rightarrow u^{p} \quad \mu$-a.e., by the martingale convergence theorem. This completes the proof. 
(4.8) Definition. For each $n \geq 1$, let $u_{n}$ and $v_{n}$ be as defined in the hypothesis of the previous lemma. Define

$$
Q^{n}: L_{p}\left(X, \mathscr{F}_{n}, \mu_{n}\right) \rightarrow L_{p}(d \omega),
$$

where $\mu_{n}$ is the restriction of $\mu$ to $\mathscr{F}_{n}$, by

$$
Q^{n} f=\frac{v_{n}}{u_{n} \circ \pi}(f \circ \pi)
$$

for $f \in L_{p}\left(X, \mathscr{F}_{n}, \mu_{n}\right)$.

By Lemma (4.6), this is an isometry. If $\omega_{n}$ is the restriction of $\omega$ to $\mathscr{I}_{n}$, and we make the obvious identification of $\left(W, \mathscr{I}_{n}, \omega_{n}\right)$ with $\left(X, \mathscr{F}_{n}, \mu_{n}\right)$, then define $T^{n}: L_{p}(d \mu) \rightarrow L_{p}\left(X, \mathscr{F}_{n}, \mu_{n}\right)$ by

$$
T^{n} f=E\left(Q^{n} E\left(f \mid \mathscr{F}_{n}\right) \mid \mathscr{I}_{n}\right)
$$

for $f \in L_{p}(\mathbf{X})$. Each $T^{n}$ is a positive contraction, and it is easy to see that if $f \in L_{p}(d \mu)$, then $T^{n} f \rightarrow T f \mu$-a.e.

Observe that $u_{n}$ is a semi-invariant function for each $T^{n}$; the reason is that $v_{n}$ is already $\mathscr{I}_{n}$-measurable. (In fact, it is easy to see that $u_{n}$ is the only normalized semi-invariant function for $T^{n}$.) Thus, the induced operator $\left(T^{n}\right)_{r}$ is defined for any $r, 1<r<\infty$. For brevity, denote it $R_{n}$.

(4.9) Theorem. $\left\|R_{n} f-T_{r} f\right\|_{r} \rightarrow 0$ as $n \rightarrow \infty$, for every $f \in L_{r}(d \mu)$.

Proof. If $f$ is $\mathscr{F}_{n}$-measurable, then

$$
\begin{aligned}
R_{n} f & =v_{n}^{p / r-1} T^{n}\left(v_{n}^{1-p / r} f\right) \\
& =v_{n}^{p / r-1} E\left[\frac{v_{n}}{u_{n} \circ \pi}\left(u_{n}^{1-p / r} \circ \pi\right)(f \circ \pi) \mid \mathscr{I}_{n}\right] \\
& =E\left[\left(\frac{v_{n}}{u_{n} \circ \pi}\right)^{p / r}(f \circ \pi) \mid \mathscr{I}_{n}\right],
\end{aligned}
$$

since $v_{n}$ is $\mathscr{I}_{n}$-measurable. Whether or not $f$ is $\mathscr{F}_{n}$-measurable, define

$$
\phi_{n}=\left(\frac{v_{n}}{u_{n} \circ \pi}\right)^{p / r}\left[E\left(f \mid \mathscr{F}_{n}\right) \circ \pi\right]
$$

Then $R_{n} f=E\left(\phi_{n} \mid \mathscr{I}_{n}\right)$ for any $f \in L_{r}(d \mu)$. Similarly, if $\phi=(v / u \circ \pi)^{p / r}(f \circ \pi)$, then $T_{r} f=E(\phi \mid \mathscr{J})$.

Clearly $\phi_{n} \rightarrow \phi$ a.e.; if we can show that $\left\|\phi_{n}\right\|_{r} \rightarrow\|\phi\|_{r}$, we may conclude that $\phi_{n} \rightarrow \phi$ in $L_{r}$ norm (see [Ry, p. 118]):

$$
\begin{aligned}
\left\|\phi_{n}\right\|_{r}^{r} & =\left\|Q^{n}\left(\left[E\left(f \mid \mathscr{F}_{n}\right)\right]^{r / p}\right)\right\|_{p}^{p} \\
& =\left\|\left[E\left(f \mid \mathscr{F}_{n}\right)\right]^{r / p}\right\|_{p}^{p}=\left\|E\left(f \mid \mathscr{F}_{n}\right)\right\|_{r}^{r} \rightarrow\|f\|_{r}^{r},
\end{aligned}
$$

as $E\left(f \mid \mathscr{F}_{n}\right)$ is an $L_{r}$ martingale. The second line follows because $Q^{n}$ is an isometry. Also $\|\phi\|_{r}=\|f\|_{r}$ by a similar calculation. This tells us that

$$
\left\|\phi_{n}-\phi\right\|_{r} \rightarrow 0
$$


To conclude the proof, observe that

$$
\left\|R_{n} f-T_{r} f\right\|_{r} \leq\left\|E\left(\phi_{n} \mid \mathscr{F}_{n}\right)-E\left(\phi_{n} \mid \mathcal{F}\right)\right\|_{r}+\left\|E\left(\phi_{n} \mid \mathcal{F}\right)-E(\phi \mid \mathscr{I})\right\|_{r} .
$$

The first term tends to zero by the martingale convergence theorem and the second term is dominated by $\left\|\phi_{n}-\phi\right\|_{r}$.

\section{Convergence of the ALTeRnating SEQUeNCE}

This section is in many ways analogous to $\S \S 3$ and 4 of [AS2], and so the reader will often be referred there for details. Where we follow [AS2] closely, every effort is made to keep the notation consistent.

(5.1) Definitions. Suppose $1<p<\infty, 1<r<\infty$, and let $\psi=\psi_{p, r}$. Let $\left\langle T_{n}\right\rangle_{n=1}^{\infty}$ be a sequence of positive linear contractions with semi-invariant functions operating on the $L_{p}$ space of a $\sigma$-infinite Lebesgue space. Call such a sequence of operators a norming sequence. Call a norming sequence special if all operators are finite dimensional.

Let $V_{0}$ and $U_{0}$ be the identities on $L_{p}$ and $L_{r}$ respectively, and make the following definitions for each $n \geq 1$ :

$$
V_{n}=T_{n} \cdots T_{1}, \quad U_{n}=\left(T_{1}^{*}\right)_{r} \cdots\left(T_{n}^{*}\right)_{r} .
$$

For a given $f \in L_{p}$ and an $n \geq 0$, let $g_{n}=U_{n} \psi\left(V_{n} f\right)$. Observe that $g_{0}=\psi f$ and that $\left\|g_{0}\right\|_{r}=\|f\|_{p}^{p / r}$.

We say that Estimate A holds for a norming sequence $\left\langle T_{n}\right\rangle_{n=1}^{\infty}$ if

$$
\left\|\sup _{n \geq 0}\left|g_{n}\right|\right\|_{r} \leq\left(q\|f\|_{p}\right)^{p / r}\left(=q^{p / r}\left\|g_{0}\right\|_{r}\right)
$$

for every $f \in L_{p}$.

We say that Estimate B holds for a norming sequence $\left\langle T_{n}\right\rangle_{n=1}^{\infty}$ if for every $\varepsilon>0$ there is a $\delta>0$, depending only on $\varepsilon, p$, and $r$, such that

$$
\left\|\sup _{n \geq 0}\left|g_{n}-g_{0}\right|\right\|_{r}<\varepsilon\|f\|_{p}^{p / r} \quad\left(=\varepsilon\left\|g_{0}\right\|_{r}\right)
$$

whenever $f \in L_{p}$ is such that

$$
\|f\|_{p}-\lim _{n \geq 0}\left\|V_{n} f\right\|_{p}<\delta\|f\|_{p} .
$$

Given a norming sequence $\left\langle T_{n}\right\rangle_{n=1}^{\infty}$, a fixed $n \geq 1$, and a function $f \in L_{p}$, let $\tilde{f}=V_{n} f$. For every $k \geq 1$, let $\widetilde{T}_{k}=T_{n+k}$. Let $\widetilde{V}_{0}$ and $\widetilde{U}_{0}$ be the identities on $L_{p}$ and $L_{r}$ respectively. For each $k \geq 1$, let

$$
\widetilde{V}_{k}=\widetilde{T}_{k} \cdots \widetilde{T}_{1}, \quad \widetilde{U}_{k}=\left(\widetilde{T}_{1}^{*}\right)_{r} \cdots\left(\widetilde{T}_{k}^{*}\right)_{r},
$$

and for each $k \geq 0$, let $\tilde{g}_{k}=\widetilde{U}_{k} \psi\left(\widetilde{V}_{k} \tilde{f}\right)$. Observe that $g_{n+k}=U_{n} \tilde{g}_{k}$ for every $k \geq 0$. 
(5.2) Theorem. Suppose Estimates A and B are satisfied for every norming sequence. Then given a norming sequence $\left\langle T_{n}\right\rangle_{n=1}^{\infty}$ and an $f \in L_{p},\left\langle g_{n}\right\rangle_{n=0}^{\infty}$ converges a.e.

Proof. Because of Estimate A, ( $\left.\sup _{n \geq 0}\left|g_{n}\right|\right) \in L_{r}$. Therefore, by Lemma (2.27), it suffices to show that

$$
\lim _{n \geq 0}\left\|\sup _{k \geq n}\left|g_{n+k}-g_{n}\right|\right\|_{r}=0 \text {. }
$$

Let $\beta=\lim _{n \geq 0}\left\|V_{n} f\right\|_{p}$ and distinguish two cases:

Case 1: $\beta=0$. Given $\varepsilon>0$, find $n_{0} \geq 1$ such that

$$
\left\|V_{n_{0}} f\right\|_{p}<\frac{1}{q}\left(\frac{\varepsilon}{2}\right)^{r / p}
$$

Fix $n \geq n_{0}$ and define $\tilde{f}$ and $\tilde{g}_{k}$ as above. Observe that $\|\tilde{f}\|_{p} \leq\left\|V_{n_{0}} f\right\|_{p}$. We have

$$
\begin{aligned}
\left\|\sup _{k \geq 0}\left|g_{n+k}-g_{n}\right|\right\|_{r} & =\left\|\sup _{k \geq 0}\left|U_{n} \tilde{g}_{k}-U_{n} \tilde{g}_{0}\right|\right\|_{r} \\
& \leq\left\|\sup _{k \geq 0}\left|\tilde{g}_{k}-\tilde{g}_{0}\right|\right\|_{r} \leq 2\left\|\sup _{k \geq 0}\left|\tilde{g}_{k}\right|\right\|_{r} \\
& \leq 2\left(q\|\tilde{f}\|_{p}\right)^{p / r}<\varepsilon,
\end{aligned}
$$

where the first inequality follows from Lemma (2.24) and the third follows from Estimate A for the sequence $\left\langle\widetilde{T}_{k}\right\rangle_{k=1}^{\infty}$.

Case 2: $\beta>0$. Given $\varepsilon>0$, choose $\delta>0$ as given by Estimate $\mathrm{B}$, corresponding to $\varepsilon /\|f\|_{p}^{p / r}$. Choose $n_{0} \geq 1$ such that $\left\|V_{n_{0}} f\right\|_{p}<(1+\delta) \beta$. Fix $n \geq n_{0}$ and define $\tilde{f}$ and $\tilde{g}_{k}$ as above. Observe that $\beta \leq\|\tilde{f}\|_{p}$, since the $\left\|V_{n} f\right\|_{p}$ 's form a monotone sequence. We have

$$
\|\tilde{f}\|_{p}-\lim _{k \geq 0}\left\|\tilde{V}_{k} \tilde{f}\right\|_{p}=\left\|V_{n} f\right\|_{p}-\beta<(1+\delta) \beta-\beta \leq \delta\|\tilde{f}\|_{p} .
$$

Now apply Estimate B for $\left\langle\widetilde{T}_{k}\right\rangle_{k=1}^{\infty}$; we conclude

$$
\left\|\sup _{k \geq 0}\left|g_{n+k}-g_{k}\right|\right\|\left\|_{r} \leq\right\| \sup _{k \geq 0}\left|\tilde{g}_{k}-\tilde{g}_{0}\right|\left\|_{r}<\left(\frac{\varepsilon}{\|f\|_{p}^{p / r}}\right)\right\| \tilde{f} \|_{p}^{p / r} \leq \varepsilon,
$$

where the first inequality follows as in Case 1.

(5.3) Lemma. If Estimate A holds for every special norming sequence, then it holds for every norming sequence.

Proof. Suppose $\left\langle T_{n}\right\rangle_{n=1}^{\infty}$ is a uniform norming sequence for which Estimate A fails. Then there is a function $f \in L_{p}$ and an $n \geq 1$ such that

$$
\left\|\max _{0 \leq k \leq n}\left|g_{k}\right|\right\|_{r}>q^{p / r}\left\|g_{0}\right\|_{r}
$$


Suppose $\left\langle\mathscr{F}_{m}\right\rangle_{m=1}^{\infty}$ is a monotone sequence of finite sub- $\sigma$-algebras of $\mathscr{F}$ with $\mathscr{F}=\sigma\left(\bigcup_{m=1}^{\infty} \mathscr{F}_{m}\right)$, the smallest $\sigma$-algebra containing the algebra $\bigcup_{m=1}^{\infty} \mathscr{F}_{m}$. For each $k$ and $m, 1 \leq k \leq n$ and $m \geq 1$, let $T_{k}^{m}$ be the finite-dimensional operator as defined in (4.8). Let $f^{m}=E\left(f \mid \mathscr{F}_{m}\right)$.

Let $m \geq 1$ be arbitrary. Let $V_{0}^{m}$ and $U_{0}^{m}$ be $E\left(\cdot \mid \mathscr{F}_{m}\right)$ operating on $L_{p}$ and $L_{r}$ respectively. For each $k, 1 \leq k \leq n$, let

$$
V_{k}^{m}=T_{k}^{m} \cdots T_{1}^{m}, \quad U_{k}^{m}=\left(\left(T_{1}^{*}\right)^{m}\right)_{r} \cdots\left(\left(T_{k}^{*}\right)^{m}\right)_{r} .
$$

For $f \in L_{p}, m \geq 1$, and each $k, 0 \leq k \leq n$, let

$$
g_{k m}=U_{k}^{m} \psi\left(V_{k}^{m} f\right)=U_{k}^{m} \psi\left(V_{k}^{m} f^{m}\right) .
$$

By the martingale convergence theorem, $\lim _{m \geq 1}\left\|f-f^{m}\right\|_{p}=0$. We will show that

$$
\lim _{m \geq 1}\left\|g_{k m}-g_{k}\right\|_{r}=0
$$

as well. Therefore, by applying Lemma (2.26),

$$
\lim _{m \geq 1}\left\|\max _{0 \leq k \leq n}\left|g_{k m}\right|-\max _{0 \leq k \leq n}\left|g_{k}\right|\right\|_{r}=0 \text {. }
$$

Thus, for a suitably large integer $m_{0}$,

$$
\left\|\max _{0 \leq k \leq n}\left|g_{k m_{0}}\right|\right\|_{r}>\left(q\left\|f^{m_{0}}\right\|_{p}\right)^{p / r},
$$

since the same inequality holds for $f$ and the $g_{k}$ 's. Because $\mathscr{F}_{m_{0}}$ is finite, the operators $\left\langle T_{1}^{m_{0}}, \ldots, T_{n}^{m_{0}}\right\rangle$ are essentially finite dimensional. Therefore, they form the initial portion of a special norming sequence for which Estimate A fails, contradicting the hypothesis of the lemma.

To prove (5.4), we first prove

$$
\lim _{m \geq 1}\left\|V_{k}^{m} f-V_{k} f\right\|_{p}=0
$$

for every $k, 0 \leq k \leq n$. When $k=0$, this is simply the martingale convergence theorem. For the inductive step, observe that

$$
\left\|V_{k+1}^{m} f-V_{k+1} f\right\|_{p}=\left\|T_{k+1}^{m} V_{k}^{m} f-T_{k+1} V_{k} f\right\|_{p},
$$

where $\lim _{m \geq 1}\left\|V_{k}^{m} f-V_{k} f\right\|_{p}=0$ by the inductive hypothesis. We apply Theorem (4.9) with $r=p$ and Lemma (2.30) to conclude that

$$
\lim _{m \geq 1}\left\|V_{k+1}^{m} f-V_{k+1} f\right\|_{p}=0
$$

completing the induction.

Because of the uniform continuity of $\psi$,

$$
\lim _{m \geq 1}\left\|\psi V_{k}^{m} f-\psi V_{k} f\right\|_{p}=0
$$

for each $k, 0 \leq k \leq n$. 
We now perform another induction similar to the proof of (5.5) to show that when $g \in L_{r}$,

$$
\lim _{m \geq 1}\left\|U_{k}^{m} g-U_{k} g\right\|_{r}=0,
$$

for each $k, 0 \leq k \leq n$. This completes the proof.

(5.6) Lemma. Suppose that for every $\xi>0$, there is an $\eta>0$ depending only on $\xi, p$, and $r$ such that

$$
\left\|\max _{0 \leq k \leq n}\left|g_{k}^{\prime}-g_{0}^{\prime}\right|\right\|_{r}<\xi\left\|f^{\prime}\right\|_{p}^{p / r}
$$

whenever $\left\langle T_{n}^{\prime}\right\rangle_{n=1}^{\infty}$ is a special norming sequence, $n \geq 1$, and $f^{\prime} \in L_{p}$ is such that $\left\|f^{\prime}\right\|_{p}-\left\|V_{n}^{\prime} f^{\prime}\right\|_{p}<\eta\left\|f^{\prime}\right\|_{p}$, where $V_{n}^{\prime}$ and $g_{n}^{\prime}$ are defined exactly as $V_{n}$ and $g_{n}$ in (5.1), relative to $\left\langle T_{n}^{\prime}\right\rangle_{n=1}^{\infty}$. Then Estimate B holds for every norming sequence.

Proof. Let $\left\langle T_{n}\right\rangle_{n=1}^{\infty}$ be a norming sequence and suppose $\xi>0$ is given. Choose $\eta>0$ from the hypothesis of the lemma, corresponding to $\xi / 2$. If Estimate $B$ fails for $\left\langle T_{n}\right\rangle_{n=1}^{\infty}$, then there is a function $f \in L_{p}$ with $\|f\|_{p}-\left\|V_{n} f\right\|_{p}<\eta\|f\|_{p}$, but for which

$$
\left\|\max _{0 \leq k \leq n}\left|g_{k}-g_{0}\right|\right\|_{r}>\frac{\xi}{2}\|f\|_{p}^{p / r} .
$$

As in the proof of the previous lemma, we approximate the operators $T_{k}$ with the operators $T_{k}^{m}$ from (4.8). Define $g_{k m}$ as before, for each $m \geq 1$ and each $k, 0 \leq k \leq n$, and let $h_{k}=g_{k}-g_{0}$ and $h_{k m}=g_{k m}-g_{0 m}$ for the same set of indices. Then

$$
\left\|h_{k m}-h_{k}\right\|_{r} \leq\left\|g_{k m}-g_{k}\right\|_{r}+\left\|g_{0 m}-g_{0}\right\|_{r},
$$

and we have seen that both of these terms tend to zero as $m$ increases. Thus $\lim _{m \geq 1}\left\|h_{k m}-h_{k}\right\|_{r}=0$, and we may apply Lemma (2.26) to conclude

$$
\lim _{m \geq 1}\left\|\max _{0 \leq k \leq n}\left|g_{k m}-g_{0 m}\right|-\max _{0 \leq k \leq n}\left|g_{k}-g_{0}\right|\right\|_{r}=0 \text {. }
$$

At the same time, we have

$$
\lim _{m \geq 1}\left\|f-f^{m}\right\|_{p}=0 \quad \text { and } \quad \lim _{m \geq 1}\left\|V_{n} f-V_{n}^{m} f^{m}\right\|_{p}=0 .
$$

Thus, we may choose an $m_{0}$ sufficiently large that we maintain the relations

$$
\left\|f^{m_{0}}\right\|_{p}-\left\|V_{n}^{m_{0}}\right\|_{p}<\eta\left\|f^{m_{0}}\right\|_{p}
$$

and

$$
\left\|\max _{0 \leq k \leq n}\left|g_{k m_{0}}-g_{0 m_{0}}\right|\right\|_{r}>\frac{\xi}{2}\left\|f^{m_{0}}\right\|_{p}^{p / r} .
$$

As $\mathscr{F}_{m}$ is finite, $\left\langle T_{1}^{m_{0}}, \ldots, T_{n}^{m_{0}}\right\rangle$ form the initial portion of a special norming sequence for which the hypothesis of the lemma fails. 
We have reduced the proof of Theorem (1.2) to verifying that finitary versions of Estimates A and B hold for every special norming sequence. In order to show that this is true, we introduce a dilation of these operators similar to the one given in $[\mathrm{A}]$.

(5.7) Definitions. Let $(X, \mathscr{F}, \mu)$ be a measure space in which $\mathscr{F}$ is a finite set. Let $\left\{F_{i}\right\}_{i=1}^{d}$ be an enumeration of the atoms of $\mathscr{F}$ of positive measure. Let the indices $i$ and $j$ range through the integers $\{1, \ldots, d\}$. If $T$ is a positive operator with a semi-invariant function $u$, let $u=\sum_{i} \alpha_{i} \chi_{F_{i}}$ and $T u=$ $\sum_{i} \beta_{i} \chi_{F_{i}}$. We have $\alpha_{i}>0$ and $\beta_{i}>0$ for each $i$. Let $m_{i}=\mu\left(F_{i}\right)$ and let $a_{i j}=\omega\left[\pi^{-1} F_{i} \cap\left(F_{j} \times[0,1]\right)\right]$, with $\pi$ and $\omega$ as given in $\S 4$. Observe that $\sum_{i} a_{i j}=m_{j}$ for each $j$, and that for each $i$,

$$
\alpha_{i}^{p} m_{i}=\int_{F_{i}} u^{p} d \mu=\int_{\pi^{-1} F_{i}} v^{p} d \omega=\sum_{j} \beta_{j}^{p} a_{i j} .
$$

Let

$$
b_{i j}=\left(\frac{\beta_{j}}{\alpha_{i}}\right)^{p} \frac{a_{i j}}{m_{i}} .
$$

It is easy to verify that $\sum_{j} b_{i j}=1$. Observe also that $a_{i j}=0$ if and only if $b_{i j}=0$.

We are going to construct a set $Z$ in the coordinate plane $\mathbb{R}^{2}$ and an isometry of its $L_{p}$ space. The construction is virtually identical to the one given in [A] and used in [AS2], except that some of the subrectangles may have measure zero. However, because of the last observation, this will cause no problems.

Let $\left\langle I_{i}\right\rangle_{i=1}^{d}$ be disjoint intervals on the $x$-axis of the coordinate plane, each of length $m_{i}$. Let $\left\langle J_{i}\right\rangle_{i=1}^{d}$ be disjoint intervals on the $y$-axis, each of unit length. Let $P_{i}=I_{i} \times J_{i}$ and $Z=\bigcup_{i} P_{i}$. Let $\mathbb{Z}=(Z, \mathscr{B}, \lambda)$, where $\mathscr{B}$ is the Borel $\sigma$-algebra of $Z$ and $\lambda$ is the restriction of Lebesgue measure on $\mathbb{R}^{2}$ to $Z$. let $L_{p}$ denote $L_{p}(\mathbf{Z})$, and let $\mathscr{P}$ be the partition $\left\{P_{i}\right\}_{i=1}^{d}$ of $Z$. Let $E=E(\cdot \mid \mathscr{P})$ and let $l_{p}=E L_{p}$.

Define a further partitioning of $Z$ as follows. Each $I_{j}$ is partitioned into $d$ subintervals $\left\langle I_{i j}\right\rangle_{i=1}^{d}$, each of length $a_{i j}$. Each $J_{i}$ is partitioned into $d$ subintervals $\left\langle J_{i j}\right\rangle_{j=1}^{d}$, each of length $b_{i j}$. Let $R_{i j}=I_{i} \times J_{i j}$, a horizontal strip of $P_{i}$, and $S_{i j}=I_{i j} \times J_{j}$, a vertical strip of $P_{j}$.

Define a point transformation $\tau: Z \rightarrow Z$ by mapping each $R_{i j}$ of nonzero measure to the corresponding $S_{i j}$, in such a way that the Radon-Nikodým derivative for the mapping of these rectangles is constant. Thus, $\tau$ "squeezes" the width of $R_{i j}$ from $m_{i}$ to $a_{i j}$ and "stretches" its height from $b_{i j}$ to 1 ; this deformation determines the constant value of

on $S_{i j}$.

$$
\rho=\frac{d\left(\lambda \circ \tau^{-1}\right)}{d \lambda}
$$


$\lambda\left(R_{i j}\right)=0$ if and only if $\lambda\left(S_{i j}\right)=0$, because of the corresponding property of $a_{i j}$ and $b_{i j}$, and so $\tau$ is an automorphism of $Z$. An automorphism of $Z$ determined in this manner by any pair of sequences of $a_{i j}$ 's and $b_{i j}$ 's satisfying $\sum_{i} a_{i j}=m_{j}, \sum_{j} b_{i j}=1$, and $a_{i j}=0$ if and only if $b_{i j}=0$, is called an $a d-$ missible automorphism. Each admissible automorphism induces an admissible $L_{p}$ isometry $Q$ in the usual manner by $Q f=\rho^{1 / p}\left(f \circ \tau^{-1}\right)$.

(5.8) Theorem. The action of $E Q$ on $l_{p}$ is isomorphic to the action of the original operator $T$ on $L_{p}(\mathbf{X})$.

Proof. Let $i$ range through $\{1, \ldots, d\}$. Let $\Phi$ be given by

$$
\sum_{i} c_{i} \chi_{P_{i}} \in l_{p} \mapsto \sum_{i} c_{i} \chi_{F_{i}} \in L_{p}(\mathbf{X}) \text {. }
$$

This is an isometric isomorphism since $\lambda\left(P_{i}\right)=\mu\left(F_{i}\right)=m_{i}$.

Let $\mathbf{W}=(W, \mathscr{K}, \omega), \pi, \mathscr{I}$, and $v$ be as given in Theorem (4.5). According to that theorem, if we define $R: L_{p}(d \mu) \rightarrow L_{p}(d \omega)$ by

$$
R g=\frac{v}{u \circ \pi}(g \circ \pi)
$$

then $T g=E(R g \mid \mathcal{I})$ for every $g \in L_{p}(d \mu)$. Since $u=\sum_{i} \alpha_{i} \chi_{F_{i}}$ and $T u=$ $\sum_{i} \beta_{i} \chi_{F_{i}}$, we have $R g=\left(\beta_{j} / \alpha_{i}\right) c_{i}$ on each $\pi^{-1} F_{i} \cap\left(F_{j} \times[0,1]\right) \subseteq F_{j} \times I$.

When $f \in l_{p}$, then $Q f=\rho_{i j}^{1 / p} c_{i}$ on each $S_{i j} \subseteq P_{j}$, where $\rho_{i j}$ is the constant value of the Radon-Nikodým derivatives $\rho$ on the rectangle $S_{i j}$. Observe that

$$
\rho_{i j}=\frac{\lambda\left(R_{i j}\right)}{\lambda\left(S_{i j}\right)}=\frac{m_{i} b_{i j}}{a_{i j}}=\left(\frac{\beta_{j}}{\alpha_{i}}\right)^{p} .
$$

We also have $\omega\left[\pi^{-1} F_{i} \cap\left(F_{j} \times[0,1]\right)\right]=\lambda\left(S_{i j}\right)=a_{i j}$. This means that $Q f$ and $R g$ are simple functions taking the same range of values over sets of identical measure. Therefore, $T g=E(R g \mid \mathscr{F})=\Phi(E Q f)$ as desired.

The proof of the convergence of the alternating sequence is now reduced to an examination of the actions of admissible isometries of $\mathbf{Z}$, intertwined with the conditional expectation operator with respect to $\mathscr{P}$.

(5.9) Definitions. Let $G$ be a subset $\mathbb{R}^{2}$. A subset $F$ of $G$ is called a vertical subset of $G$ if

$$
F=\left(F^{\prime} \times \mathbb{R}\right) \cap G
$$

for some subset $F^{\prime}$ of the $x$-axis. Similarly, if

$$
H=\left(\mathbb{R} \times H^{\prime}\right) \cap G
$$

for some subset $H^{\prime}$ of the $y$-axis, then $H$ is called a horizontal subset of $G$.

We say that a function $f$ is constant on vertical lines if $f\left(x_{1}, y_{1}\right)=f\left(x_{2}, y_{2}\right)$ whenever $x_{1}=x_{2}$. We say that $f$ is constant on horizontal lines if $f\left(x_{1}, y_{1}\right)=$ $f\left(x_{2}, y_{2}\right)$ whenever $y_{1}=y_{2}$.

The following is a summary of Lemmas (4.5) through (4.12) from [AS2]. 
(5.10) Lemma. Let $\tau$ be an admissible automorphism, and let $Q$ be the induced $L_{p}$ isometry.

(a) Suppose $\mathscr{G}$ is a finite partition of $Z$ in which each atom is a vertical subset of some $P_{i}$. Let $f$ be an $L_{p}$ function which is constant on vertical lines. Then

$$
Q E(f \mid \mathscr{G})=E(Q f \mid \mathscr{P} \vee \tau \mathscr{G}) .
$$

$\left(a^{\prime}\right)$ Suppose $\mathscr{H}$ is a finite partition of $Z$ in which each atom is a horizontal subset of some $P_{i}$. Let $f$ be an $L_{p}$ function which is constant on horizontal lines. Then

$$
Q^{-1} E(f \mid \mathscr{H})=E\left(Q^{-1} f \mid \mathscr{P} \vee \tau \mathscr{H}\right) .
$$

(b) If $f_{1}$ and $f_{2}$ are $L_{p}$ functions that are constant on vertical lines and $E f_{1}=E f_{2}$, then also $E Q f_{1}=E Q f_{2}$.

$\left(\mathrm{b}^{\prime}\right)$ If $f_{1}$ and $f_{2}$ are $L_{p}$ functions that are constant on horizontal lines and $E f_{1}=E f_{2}$, then also $E Q^{-1} f_{1}=E Q^{-1} f_{2}$.

(c) If $f$ is constant on vertical lines, then $Q f$ is constant on vertical lines.

(c') If $f$ is constant on horizontal lines, then $Q^{-1} f$ is constant on horizontal lines.

(5.11) Definitions. Let $n$ be a fixed integer, $n \geq 1$, and let $k$ range through $\{0,1, \ldots, n\}$. If $1 \leq k \leq n$, let $\tau_{k}$ be an admissible isometry of $Z$, let $Q_{k}$ be the $L_{p}$ isometry induced by $\tau_{k}$, and let $R_{k}$ be the $L_{r}$ isometry induced by $\tau_{k}^{-1}$. Let $Q_{0}$ and $R_{0}$ be the identities on $L_{p}$ and $L_{r}$ respectively. Let

$$
\begin{array}{lll}
T_{k}=E Q_{k} E, & V_{k}=T_{k} \cdots T_{0}, & W_{k}=Q_{k} \cdots Q_{0}, \\
S_{k}=E R_{k} E, & U_{k}=S_{0} \cdots S_{k}, & D_{k}=R_{0} \cdots R_{k} .
\end{array}
$$

Observe that $S_{k}=\left(T_{k}^{*}\right)_{r}$ by Lemma (3.20).

Let $f$ be a fixed but arbitrary function in $L_{p}$. Let $g_{k}=U_{k} \psi\left(V_{k} f\right)$ and $\phi_{k}=W_{k}^{-1} E W_{k} E f$. Observe that $g_{0}=\psi \phi_{0}=\psi E f$.

(5.12) Lemma. For any $f \in L_{p}, V_{k} f=E W_{k} E f$.

Proof. This is Lemma (4.14) of [AS2]. When $k=0$ this is immediate from the definitions. The inductive step is given by Lemma $(5.10)(b)$ and (c).

(5.13) Lemma. For any $g \in L_{r}, U_{k} g=E D_{k} E g$.

Proof. We will show that

$$
S_{i} \cdots S_{j} g=E R_{i} \cdots R_{j} E g
$$

for every pair $i, j$ with $0 \leq i \leq j \leq n$. This will prove the lemma, since the desired identity is (5.14) with $i=0$ and $j=k$. The proof is by induction on $j-i$. When $i=j,(5.14)$ is simply the definition of $S_{i} g$. 
Now suppose (5.14) holds for some pair $i+1, j+1$ with $0 \leq i \leq j<n$. We have

$$
E R_{i+1} \cdots R_{j+1} E g=E S_{i+1} \cdots S_{j+1} g
$$

by the inductive hypothesis and the idempotence of $E$, the outermost operator in $S_{i+1} \cdot R_{i+1} \cdots R_{j+1} E g$ is constant on horizontal lines, by repeated application of Lemma $(5.10)\left(c^{\prime}\right)$. Thus, by Lemma $(5.10)\left(b^{\prime}\right)$, we have

$$
E R_{i} R_{i+1} \cdots R_{j+1} E g=E R_{i} S_{i+1} \cdots S_{j+1}=S_{i} \cdots S_{j+1} g .
$$

This completes the induction.

(5.15) Lemma. $g_{k}=E \psi\left(\phi_{k}\right)$.

Proof.

$$
\begin{aligned}
g_{k} & =U_{k} \psi\left(V_{k} f\right)=E D_{k} E \psi\left(E W_{k} E f\right) \\
& =E D_{k} \psi\left(E W_{k} E f\right)=E \psi\left[\left(R_{0}\right)^{p} \cdots\left(R_{k}\right)^{p} E W_{k} E f\right] .
\end{aligned}
$$

The second line follows from the two previous lemmas. The third line follows because $\psi$ maps $\mathscr{P}$-measurable functions to $\mathscr{P}$-measurable functions. For the fourth line, we use $\left(R_{i}\right)^{p}$ to denote the $L_{p}$ isometry induced by $\tau_{i}^{-1}$. Thus, this line follows by an application of Lemma (2.19). By Lemma (2.17), that isometry is $Q_{i}^{-1}$. Thus

$$
g_{k}=E \psi\left(W_{k}^{-1} R W_{k} E f\right)=E \psi\left(\phi_{k}\right),
$$

as desired.

(5.16) Lemma. There exists a monotone sequence $\mathscr{G}_{n} \subseteq \mathscr{G}_{n-1} \subseteq \cdots \subseteq \mathscr{G}_{0}$ of finite $\sigma$-algebras such that

$$
\phi_{k}=W_{n}^{-1} E\left(W_{n} E f \mid \mathscr{G}_{k}\right) \text {. }
$$

Proof. This is Lemma (4.16) of [AS2]. We may take $\mathscr{G}_{n}=\mathscr{P}$. Lemma (5.10)(a) provides the induction step needed to show that we may take

$$
\mathscr{G}_{n-k}=\mathscr{P} \vee \tau_{n} \mathscr{P} \vee \cdots \vee \tau_{n} \cdots \tau_{n-k+1} \mathscr{P}
$$

when $1 \leq k \leq n$.

(5.17) Definition. Let $u_{k}=E\left(W_{n} E f \mid \mathscr{G}_{k}\right)$, where the $\mathscr{G}_{k}$ 's are as in the previous lemma. Observe that $\phi_{k}=W_{n}^{-1} u_{k}$.

(5.18) Theorem. The sequence $\left\langle u_{0}, \ldots, u_{n}\right\rangle$ is an $L_{p}$ martingale. Furthermore,

$$
\left\|\max _{0 \leq k \leq n}\left|u_{k}\right|\right\|_{p} \leq q\left\|u_{0}\right\|_{p}
$$

and

Proof.

$$
\left\|\max _{0 \leq k \leq n}\left|u_{k}-u_{n}\right|\right\|_{p} \leq q\left\|u_{0}-u_{n}\right\|_{p} \text {. }
$$

$$
u_{k}=E\left(W_{n} E f \mid \mathscr{G}_{k}\right)=E\left(E\left(W_{n} E f \mid \mathscr{G}_{0}\right) \mid \mathscr{G}_{k}\right)=E\left(u_{0} \mid \mathscr{G}_{k}\right),
$$


since $\mathscr{G}_{k} \subseteq \mathscr{G}_{0}$ for every $k, 0 \leq k \leq n$. As well,

$$
u_{k}-u_{n}=E\left(u_{0} \mid \mathscr{G}_{k}\right)-E\left(u_{n} \mid \mathscr{G}_{k}\right)=E\left(u_{0}-u_{n} \mid \mathscr{G}_{k}\right) \text {. }
$$

In the first case, this follows from the above computation. In the second case, $u_{n}=E\left(u_{n} \mid \mathscr{G}_{k}\right)$ because $u_{n}$ is already constant on the atoms of $\mathscr{G}_{k}$.

The lemma now follows by an application of the martingale convergence theorem for $L_{p}$.

(5.19) Theorem. $\left\|\max _{0 \leq k \leq n}\left|g_{k}\right|\right\|_{r} \leq\left(q\|f\|_{p}\right)^{p / r}$.

Proof. Since $\phi_{k}=W_{n}^{-1} u_{k}$ and $W_{n}^{-1}$ is a positive isometry, we have $\left|\phi_{k}\right|=$ $W_{n}^{-1}\left|u_{k}\right|$ and $\max _{0 \leq k \leq n}\left|\phi_{k}\right|=W_{n}^{-1}\left(\max _{0 \leq k \leq n}\left|u_{k}\right|\right)$ and so

$$
\left\|\max _{0 \leq k \leq n}\left|\phi_{k}\right|\right\|_{p}=\left\|\max _{0 \leq k \leq n}\left|u_{k}\right|\right\|_{p} \leq q\left\|u_{0}\right\|_{p} \leq q\|f\|_{p} .
$$

The inequalities follow by an application of Theorem (5.18) and the fact that $\left\|u_{0}\right\|_{p}=\|E f\|_{p}$.

Since $g_{k}=E \psi\left(\phi_{k}\right)$, we have

$$
\max _{0 \leq k \leq n}\left|g_{k}\right| \leq E\left(\max _{0 \leq k \leq n}\left|\psi\left(\phi_{k}\right)\right|\right)=E \psi\left(\max _{0 \leq k \leq n}\left|\phi_{k}\right|\right),
$$

where Lemma (2.24) was used for the inequality. Thus

$$
\begin{aligned}
\left\|\max _{0 \leq k \leq n}\left|g_{k}\right|\right\|_{r} & \leq\left\|\psi\left(\max _{0 \leq k \leq n}\left|\phi_{k}\right|\right)\right\|_{r} \\
& =\left\|\max _{0 \leq k \leq n}\left|\phi_{k}\right|\right\|_{p}^{p / r} \leq\left(q\|f\|_{p}\right)^{p / r} .
\end{aligned}
$$

(5.21) Theorem. For any $\xi>0$ there is an $\eta>0$ depending only on $\xi$, $p$, and $r$ such that

$$
\left\|\max _{0 \leq k \leq n}\left|g_{k}-g_{0}\right|\right\|_{r}<\xi\|f\|_{p}^{p / r}
$$

whenever $\|f\|_{p}-\left\|V_{n} f\right\|_{p}<\eta\|E f\|_{p}$.

Proof. Since $u_{n}=E\left(u_{0} \mid \mathscr{G}_{n}\right)$, we may apply Lemma (2.25) to choose an $\eta>0$, depending only on $\delta$ (which will be specified later) and $p$ so that

$$
\left\|u_{0}\right\|_{p}-\left\|u_{n}\right\|_{p}<\eta\left\|u_{0}\right\|_{p}
$$

implies

$$
\left\|u_{0}-u_{n}\right\|_{p}<\frac{\delta}{2 q}\left\|u_{0}\right\|_{p} .
$$

We have already observed that $\left\|u_{0}\right\|_{p}=\|E f\|_{p}$. As well, we note that $\left\|u_{n}\right\|_{p}=$ $\left\|V_{n} f\right\|_{p}$. Thus, if $\|f\|_{p}-\left\|V_{n} f\right\|_{p}<\eta\|E f\|_{p}$, we have

$$
\begin{aligned}
\left\|\max _{0 \leq k \leq n}\left|u_{k}-u_{0}\right|\right\|_{p} & \leq 2\left\|\max _{0 \leq k \leq n}\left|u_{k}-u_{n}\right|\right\|_{p} \\
& \leq 2 q\left\|u_{0}-u_{n}\right\|_{p}<\xi\left\|u_{0}\right\|_{p},
\end{aligned}
$$

where the second inequality follows from Theorem (5.18). 
As in the proof of the previous theorem, we deduce

$$
\max _{0 \leq k \leq n}\left|\phi_{k}-\phi_{0}\right|=W_{n}^{-1}\left(\max _{0 \leq k \leq n}\left|u_{k}-u_{0}\right|\right)
$$

and so $\left\|\max _{0 \leq k \leq n}\left|\phi_{k}-\phi_{0}\right|\right\|_{p} \leq \delta\|E f\|_{p}$.

Since the inequality $\left\|\max \left|\phi_{k}\right|\right\|_{p} \leq q\|E f\|_{p}$ is simply a restatement of (5.20), we are in a position to apply Lemma (2.29). Choose $\delta$ from that lemma corresponding to $\xi, q$ (which depends only on $p$ ), $p$ and $r$, and conclude that

$$
\left\|\max _{0 \leq k \leq n}\left|\psi\left(\phi_{k}\right)-\psi\left(\phi_{0}\right)\right|\right\|_{r}<\xi\|E f\|_{p}^{p / r}
$$

whenever $\|f\|_{p}-\left\|V_{n} f\right\|_{p}<\eta\|E f\|_{p}$.

Now apply Lemma (2.24):

$$
\begin{aligned}
\left\|\max _{0 \leq k \leq n}\left|g_{k}-g_{0}\right|\right\|_{r} & \leq\left\|E\left(\max _{0 \leq k \leq n}\left|\psi\left(\phi_{k}\right)-\psi\left(\phi_{0}\right)\right|\right)\right\|_{r} \\
& <\xi\|E f\|_{p}^{p / r} .
\end{aligned}
$$

This completes the proof of this theorem, and hence of Theorem (1.2).

\section{REFERENCES}

[A] M. A. Akcoglu, A pointwise ergodic theorem in $L_{p}$-spaces, Canad. J. Math. 27 (1975), 1075-1082.

[AK] M. A. Akcoglu and P. E. Kopp, Construction of dilations of positive $L_{p}$-contractions, Math. Z. 155 (1977), 119-127.

[AS1] M. A. Akcoglu and L. Sucheston, On positive dilations to isometries in $L_{p}$ spaces, in Measure Theory Oberwohlfach 1975, Lecture Notes in Math., vol. 541, Springer-Verlag, New York, 1976.

[AS2] _ Pointwise convergence of alternating sequences, Canada. J. Math. 40 (1988), 610-632.

[K1] C. H. Kan, On Fong and Sucheston's property of operators in a Hilbert space, Acta Sci. Math. 41 (1979), 317-325.

[K2] _ Norming vectors of linear operators between $L_{p}$-spaces, Pacific J. Math. (to appear).

[M] S. Mazur, Une remarque sur l'homéomorphie des champs fonctionels, Studia Math. 1 (1929), 83-85.

[Rt] G. C. Rota, An “Alternierende Verfahren" for general positive operators, Bull Amer. Math. Soc. 68 (1962), 95-102.

[Ry] H. L. Royden, Real analysis, Macmillan, New York, 1968.

[S] E. M. Stein, Topics in harmonic analysis, Princeton Univ. Press, Princeton, N. J., 1970.

Department of Mathematics, University of Toronto, Toronto, Ontario M5S 1A1, Canada (Current address of M. A. Akcoglu)

Current address (R. E. Bradley): Department of Mathematics, Northwestern University, Evanston, Illinois 60209 